\title{
Anticholinergic Burden and Cognitive Performance in Patients With Schizophrenia: A Systematic Literature Review
}

\author{
Rafaella Georgiou, Demetris Lamnisos and Konstantinos Giannakou* \\ Department of Health Sciences, School of Sciences, European University Cyprus, Nicosia, Cyprus
}

Objective: Cognitive impairment in schizophrenia forms the key cause of the disease's disability, leading to serious functional, and socioeconomic implications. Dopaminergic-cholinergic balance is considered essential to cognitive performance in schizophrenia and patients are often treated with many drugs with anticholinergic properties. This study aims to examine the cognitive impact of anticholinergic burden in patients with schizophrenia.

Methods: A systematic literature review was performed on English-language studies published on PubMed, Embase, and Web of Science, from inception to June 2021, to identify research studies that examined the effect of anticholinergic load on cognition

OPEN ACCESS

Edited by:

Kelly Anne Allott,

University of Melbourne, Australia

Reviewed by:

Hiroyoshi Takeuchi,

Keio University, Japan

Stefano Barlati,

University of Brescia, Italy

*Correspondence: Konstantinos Giannakou

K.Giannakou@euc.ac.cy

Specialty section: This article was submitted to Schizophrenia,

a section of the journal

Frontiers in Psychiatry

Received: 19 September 2021

Accepted: 21 October 2021

Published: 28 December 2021

Citation:

Georgiou R, Lamnisos D and Giannakou K (2021) Anticholinergic Burden and Cognitive Performance in Patients With Schizophrenia: A Systematic Literature Review. Front. Psychiatry 12:779607. doi: 10.3389/fpsyt.2021.779607 in clinically stable patients with schizophrenia. No restrictions on study design, age of participants, or geographical distribution were applied. Two researchers performed independently the screening and shortlisting of the eligible articles. A narrative synthesis of the main characteristics and findings of studies included was reported.

Results: In total, 17 articles of varying methodological design met the inclusion criteria. Three of them found statistically significant improvement in cognition after anticholinergic tapering without adverse effects. Thirteen studies found a statistically significant association between high anticholinergic burden and cognitive impairment (neurocognitive composite scores and individual cognitive domains such as learning and memory, executive function, processing speed), apart from a study, related to the specific characteristics of clozapine.

Conclusions: Medication with increased anticholinergic load has been found in most of the studies to negatively affect neurocognitive performance of patients with schizophrenia. However, the clinical and methodological heterogeneity of studies included limit our interpretation and conclusions.

Keywords: cognitive function, anticholinergic burden, schizophrenia, psychopharmacology, systematic review

\section{INTRODUCTION}

Cholinergic neurotransmission plays a crucial role in both psychotic symptoms and cognitive disorders as well as treatment of schizophrenia (1). Specifically, an increased number of cholinergic neurons in the reticular formation of some patients with schizophrenia has been reported (2). Additionally, studies have found a decrease in muscarinic receptor levels in brain regions critical to 
the pathophysiology of schizophrenia (e.g., frontal cortex, basal ganglia, and hippocampus) (3-5) and particularly the alteration of M1 muscarinic receptors has been significantly associated with memory and learning deficits observed in the disease (6). Furthermore, studies of post-mortem brains from patients with schizophrenia, which exhibit a reduction of $\alpha 7$ $\mathrm{nAChR}$ expression in the hippocampus and cingulate cortex $(7,8)$ as well as genome-wide association studies that relate the risk for schizophrenia with the copy number variations of a locus containing the $\alpha 7 \mathrm{nAChR}$, reveal the abnormal cholinergic regulation of the disease (9). The high smoking rates observed in schizophrenia may also be due to patients using nicotine, an nAChRs agonist, as a self-medication (10, 11). It is also worth noting that literature indicates a tight link between mesolimbic dopaminergic and basal forebrain cholinergic activity (12-14) and therefore this interaction may explain the integration of motivational functions with attentional functions. Abnormal mesolimbic dopaminergic activity is likely to alter cholinergic function and thus attentional performance, which is also supported by rodent models of attention impairment. According to Kozak's et al. findings (15), animals with sensitized mesolimbic dopaminergic functions exhibited cholinergic systems that remain "frozen" at baseline and unable to support attentional performance, while a different animal study showed that stimulation of mesolimbic-corticopetal cholinergic circuitry enhanced attention performance (16). Thus, increased understanding of cholinergic neurotransmission of cortical function can contribute particularly to the understanding of attentional dysfunction observed in schizophrenia.

In addition to findings that correlate the pathogenetic mechanism of the disease with abnormal cholinergic neurotransmission, patients with schizophrenia are often treated in clinical practice with anticholinergic drugs to control the extrapyramidal side effects that cause most antipsychotics, especially first generation (17-19). Furthermore, most of psychotropic medications used have, beyond the affinity of D2 dopamine receptors, also anticholinergic activity (19). Although antipsychotics may improve clinical symptoms of patients with schizophrenia, high doses or a combination of different types of antipsychotic drugs have been associated with a decrease in cognitive functions $(20,21)$. Tani et al. (22) argue that due to the disturbance of the cholinergic system in schizophrenia, any exogenous anticholinergic activity can cause endogenous anticholinergic activity and therefore the appearance and exacerbation of the symptoms of the disease. While there are many hypotheses about how antipsychotics are associated with the decline in cognitive abilities of patients with schizophrenia, there is evidence to suggest that the cumulative anticholinergic effect of the various pharmacotherapies may be an important factor. Therefore, one could assume that particularly cognitive domains such as attention, working memory, and spatial memory, which according to the literature are affected by cholinergic regulation may also be adversely affected by the accumulated anticholinergic load of pharmacotherapies.

Given that impaired cognitive performance is a strong determinant of the outcome of the disease as well one of the major causes of the disease's disability affecting the daily social, occupational functionality, autonomy, and independence $(23,24)$, there arises the need to investigate the association of medication anticholinergic load with the cognitive deficits in patients with schizophrenia. Thus, the aim of this systematic review is to summarize the findings of epidemiological studies that assessed the effect of anticholinergic burden on cognitive performance of patients with schizophrenia and to examine the importance of these findings in future research and clinical practice.

\section{METHODS}

For the conduction of this systematic review, we followed the Preferred Reporting Items for Systematic Reviews and MetaAnalyses guidelines (PRISMA) (25).

\section{Search Strategy}

A systematic literature search in PubMed, Embase, and Web of Science was conducted by two independent authors (RG and KG) to identify articles that examined the effect of anticholinergic load on cognitive domains in patients with schizophrenia. The following terms were used: (anticholinergic OR antimuscarinic OR parasympatholytic) AND (cognitive OR brain OR memory OR dementia OR confusion) AND schizophrenia. All databases were searched from their inception through June 2021, restricted to English language publications. First, the title and abstract of each article was examined, and then the full texts of potentially eligible articles to be included in the systematic review were evaluated. A reference list of relevant studies was screened to identify additional studies.

\section{Eligibility Criteria}

We used the Population, Intervention, Comparison, Outcomes and Study design (PICOS) approach (26) for the identification of included studies. Participants were only patients with schizophrenia regardless of age and gender, while for the intervention we considered the effect of anticholinergic load medication treatment. For the comparison, studies had a control/comparison group of patients or not were eligible for inclusion. The chosen outcome was the impact of anticholinergic load on cognitive domains in people with schizophrenia. For study design, any design was eligible for inclusion.

\section{Intervention of Interest}

Assessment of exposure's degree to drugs with anticholinergic properties is a combination of the daily dose, the affinity to muscarinic receptors, the permeability to the blood barrier, and drugs' general pharmacokinetic and pharmacodynamic characteristics. Therefore, many different scales and methods of classifying cumulative anticholinergic burden are available. Eligible intervention criteria were (a) the tapering of anticholinergic therapy or (b) the cumulative anticholinergic load ranked either by anticholinergic classification scales or calculated based on serum anticholinergic activity (SAA).

Anticholinergic Drug Scale (ADS): A classification system for rating anticholinergic potency of medicines based on the clinical experience, expert opinion, and in vitro pharmacological 
properties. Medicines with anticholinergic properties are ranked from 0 (none) to 3 (high) $(27,28)$. Anticholinergic cognitive burden (ACB) scale: A classification system, which ranks drugs based on serum anticholinergic activity or in vitro binding affinity with muscarinic receptors. Drugs are graded from 0 (no anticholinergicity) to 3 (high anticholinergicity with significant adverse cognitive effects) (29). Anticholinergic burden classification (ABC): A classification system which quantifies anticholinergic burden ranging from 0 (none) to 3 (high) based on serum anticholinergic activity and expert opinion (30). SAA: Serum levels of anticholinergic drugs measured with a competitive radioreceptor binding assay technique (e.g., by Tune and Coyle) (31).

\section{Outcome of Interest}

Since cognitive impairment in schizophrenia is observed in a variety of cognitive domains (e.g., attention, working memory, verbal memory, etc.) different diagnostic tools can be used for cognitive performance evaluation. The MATRICS Consensus Cognitive Battery (MCCB), although an FDA-approved neurocognition test battery, is usually only used to extract an overall score, whereas the assessment of complex domains such as executive function requires additional diagnostic tools (e.g., Wisconsin Card Sorting Test, Stroop Test, Trail Making Test) (32). Comprehensive neuropsychological assessments are often difficult in clinical practice to carry out, so there are several brief evaluation tools, which can provide useful information on the outcome of interest (e.g., Brief Assessment of Cognition for Schizophrenia; BACS) (33). Therefore, due to the complexity of the cognitive outcome, different neurocognitive assessment batteries meet the inclusion criteria. However, studies with functional and structural imaging outcomes are beyond the scope of this article and are excluded from this systematic review because they lead to greater variability and lack of outcome coherence.

\section{Data Extraction and Quality Assessment}

Two authors (RG \& KG) extracted data independently and any discrepancy was resolved by discussion with the third author (DL). A standardized data extraction form was developed for our systematic review and it was used to minimize data entry errors and improve validity and reliability. The extracted data included first author, publication year, country of origin, design of the study, sample size, characteristics of the study population, study groups, considered confounding variables, and summary of findings.

The methodological quality of cohort and case-control studies was performed by the Newcastle-Ottawa Scale (NOS) (34). The NOS is a validated scale to assess non-randomized control trials and each study can be awarded up to nine stars. Each study is assessed on eight questions, in three groups: quality of selection, comparability between the groups and outcome. Studies with NOS values $\geq 6$ were considered moderate to high-quality studies (34). Studies with NOS values lower than 6 were considered low-quality studies. The Newcastle-Ottawa quality assessment scale has been adapted for cross-sectional studies and was used for the methodological quality of the included cross-sectional studies (35). Quality assessment of clinical trial studies was carried out using the validated modified Jadad scale $(36,37)$. Modified Jadad scale is a scoring system that allows a maximum score of 8 , assessing randomization, blinding, presentation of withdrawals/dropouts, inclusion/exclusion criteria, adverse effects, and statistical analysis. Clinical studies with a score ranging from 4 to 8 are considered moderate to high methodological quality design, whereas lower than 4 are considered low methodological design.

\section{RESULTS}

\section{Study Selection and Characteristics}

From the initial electronic search, we found a total of 734 articles, 679 of which were excluded based on title and abstract review. The full text of the remaining 55 was checked for eligibility and 38 of them were excluded. Overall, a total of 17 articles were included in this systematic review (Figure 1). No further articles were identified through searching the reference list of reviews identified during the initial search nor the reference list of the included articles. Meta-analysis was not possible to be conducted due to the heterogeneity of the data and the clinical and methodological variability of the studies included. Therefore, a narrative synthesis of the main characteristics and findings of the studies included was reported.

The publication years were from 1982 to 2021. The countries covered are the United States, China, South Korea, Germany, Canada, Japan, and Israel. The number of participants included in the studies ranged from 15 to 1,120. Most of the studies included, recruited clinically stable adults in inpatient setting, with no history of pre-existing organic impairment, unstable medical or neurological conditions, or prohibited drug abuse. As regards to the study design, the majority were cross-sectional studies (38-47). Additionally, one retrospective (19), four prospective cohort studies (48-51), and two randomized clinical trials $(52,53)$ were included. Regarding the intervention assessment method, three studies assessed cognitive performance after anticholinergic tapering, six studies evaluated the influence of serum anticholinergic activity (SAA) on cognition, and eight studies assessed the influence of medication anticholinergic burden using anticholinergic classification scales on cognitive function.

Tables 1-3 summarize the demographics of the participants, the main characteristics, and findings of the studies included accordingly to the intervention assessment method. Below are presented in detail the main results of the studies that refer to the impact of medication anticholinergic burden in specific cognitive domains evaluated with different neurocognitive tasks in patients with schizophrenia.

\section{Anticholinergic Tapering and Cognitive Performance}

The characteristics of studies included that examine the association of anticholinergic tapering with neurocognitive performance are presented in Table 1. In light of the administration of anticholinergic agents to control 


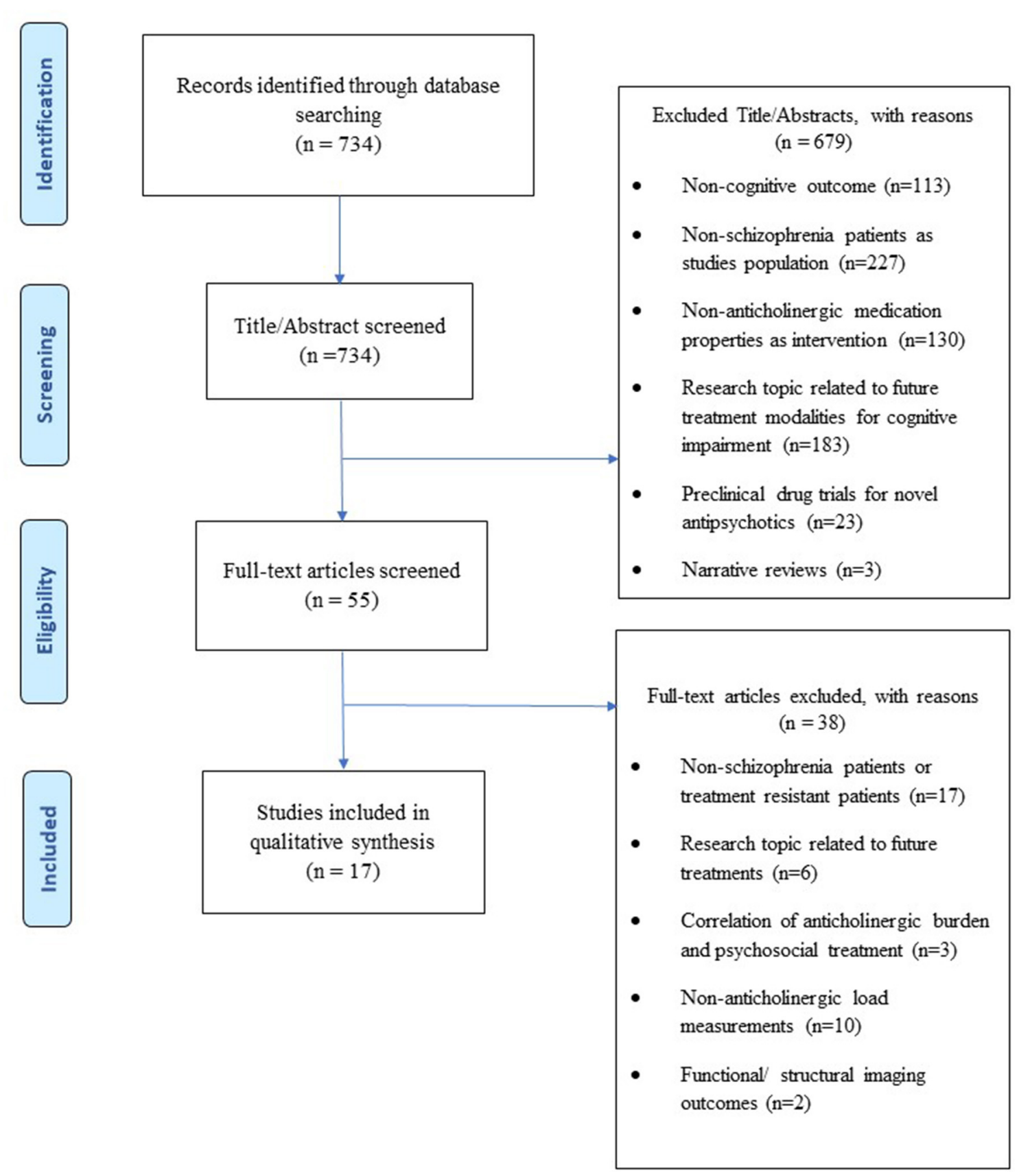

FIGURE 1 | PRISMA flow diagram of search strategy and final selection of articles.

extrapyramidal symptoms, three prospective studies (4850) examined the effect of anticholinergic tapering on cognitive functions. Despite the small number of participants $(n=27$, $n=34$, and $n=20$ ), after successful discontinuation of anticholinergic treatment by most participants, no adverse effects to psychopathology or extrapyramidal symptoms were found, while a significant improvement in the composite/overall scores of the neurocognitive batteries used (BACS, BACSJ, ADAS-Cog) during the follow-up weeks was observed.
Anticholinergic tapering showed improvement over baseline in cognitive tasks that mainly assess the domains of processing speed, attention, ideational Praxis, and Orientation.

In the Desmarais et al. (48) patients with schizophrenia or schizoaffective disorder were recruited and treated with a long-term stable dose of antipsychotics and anticholinergics $(7.3 \pm 3.3 \mathrm{mg} /$ day $)$, whereas the Ogino et al. (50) included patients with paranoid type of schizophrenia who received stable dose of second-generation antipsychotics with co-administered 
biperiden (2.2 $\pm 0.8 \mathrm{mg} /$ day $)$ as well as a control group, which neutralized the confounding factor of the practice effect. In the Desmarais et al. study (48) anticholinergic tapering revealed significant improvement in the composite $\mathrm{z}$ score of BACS at weeks 6,8 , and 12 compared to the starting point ( $p=0.029, p=0.001$, and $p=0.002$, respectively), with average corresponding differences -0.287 (95\% CI: -0.023 to $-0.552),-0.416$ (95\% CI: -0.146 to -0.686 ), and -0.517 (95\% CI: -0.163 to -0.871 ), whereas in the Ogino et al. (50) in the overall interaction (time $\times$ groups), statistical significance appeared in the composite score assessed with BACS$\mathrm{J}\left[F_{(1,32)}=6.06, p=0.02\right], 4$ weeks after discontinuation. In both studies improvement was observed in tasks that incorporate the domains of attention and processing speed. Particularly in the Desmarais et al. (48) significant improvements were observed mainly in motor tasks, in which in the 12th week the average $\mathrm{z}$ value was significantly higher than the baseline by an average difference of -0.476 ( $\mathrm{p}=0.023$ ) with $95 \%$ CI from -0.049 to -0.902 , as well as in symbol coding tasks $(p=0.043)$ with an average improvement of -0.279 and $95 \%$ CI from -0.006 to -0.552 . As regards the Ogino et al. (50), in the analysis between biperiden discontinuation group and control group, significant improvements were observed in symbol coding tasks in the anticholinergic discontinuation group $\left[F_{(1,32)}=4.75, p=0.04\right]$ and regarding the analysis among the participants of the biperiden discontinuation group, significant time changes in verbal fluency $\left[F_{(1,32)}=6.56, p=0.02\right]$ as well as in symbol coding task $\left[F_{(1,32)}=6.21, p=0.02\right]$ were observed. In the overall interaction (time $\times$ groups), also a statistical significance appeared in the symbol coding test $\left[F_{(1,32)}=10.66, p=0.003\right]$. Drimer et al. (49) used a different diagnostic tool, ADAS-Cog (Alzheimer's Disease Assessment Scale-Cognitive), with elderly patients above the age of 60 receiving biperiden ( $2-6 \mathrm{mg} /$ day) for at least 1 year. Statistically significant improvements related to previous biperiden doses were also found. Specifically, improvements were presented in the ideational Praxis subscales $(t=2.63, p<0.02)$, orientation $(t=2.41, p<0.03)$, and overall score $(t=2.43, p<0.03) 10$ days after anticholinergic discontinuation.

\section{Anticholinergic Burden and Cognitive Performance \\ Anticholinergic Burden and Composite Cognitive Scores/Global Cognition}

Out of the six studies that evaluated the estimated overall cognitive performance with the combination of representative cognitive tests from multiple domains, five provided evidence that increased medication anticholinergic burden was significantly associated with decreased global cognition or composite scores comprised of several different cognitive tests.

Specifically, in the study of Kim et al. (40), in which participants were classified into a "low ADS" and a "high ADS" group with a score of $<$ or $\geq 3$ respectively, high anticholinergic load of medication (ADS $\geq 3$ ) was associated with worse cognitive performance, assessed with MCCB (composite MCCB score, $r=-0.512, p<0.001$ ). The correlation was also confirmed through regression analysis (composite MCCB score, $\mathrm{R}^{2}=$ $0.262, p<0.001$ ). Although the explanatory power decreased after adjusted analysis, anticholinergic load continued to have a statistically negative impact on the MCCB composite score $(p=0.013)$. The recent study of Joshi et al. with a large sample size (47) was also in agreement with these findings using a different intervention and outcome assessment method. Specifically, patients with higher scores based on Anticholinergic Cognitive Burden (ACB) scale demonstrated worse cognitive performance across all cognitive domains assessed with Penn Computerized Neurocognitive Battery (PCNB) compared to patients with low ACB scores. The cross-sectional study of Eum et al. (39) detected a different threshold (ADS $\geq 4$ ) of the adverse effects of anticholinergic burden on clinically stable patients with schizophrenia. Subjects with an ADS score $\geq 4$ demonstrated statistically significant poorer cognitive composite scores $(p=0.004)$ evaluated with the BACS, by approximately a mean of 0.58 lower $\mathrm{SD}$, than patients with an ADS score $<4$. In addition, an also large study in terms of the number of participants $(n=705)$ found that the anticholinergic burden had a significant negative correlation with cognitive performance in global cognition however the effect size was small for anticholinergic burden scale (ABC), $\left(\right.$ Cohen $\left.\mathrm{f}^{2}=0.008\right)$ and for anticholinergic drug scale (ADS), (Cohen $\mathrm{f}^{2}=0.017$ ), and therefore the clinical significance of the result is not apparent (38). The different study approach of the randomized controlled clinical trial of Vinogradov et al. (53) also provided evidence that increased anticholinergic load negatively affects the response to cognitive training with the intensive auditory training "based on neuroplasticity." Increased SAA which showed a negative correlation with the improvement of global cognition in participants in audio training (Pearson's $r=-0.46, p<0.02)$ represented $20 \%$ of the variation in global cognition change, regardless of age, IQ, or severity of symptoms. On the other hand, the study of Tracy et al. (51) including 22 chronic patients with schizophrenia found no effect of the anticholinergic load on the cognitive measure. Particularly, differences in global cognition were not observed between patients with significantly higher serum anticholinergic levels $(p<0.001)$ treated with antipsychotic clozapine compared to those treated with antipsychotic risperidone (lower SAA).

\section{Anticholinergic Burden and Learning, Memory, and Verbal Skills}

In total, six studies with different methodological design and a variety of medication anticholinergicity measures reported significant association between increased anticholinergic burden and decline in several types of memory (declarative, verbal, short-term) as well as the domains of learning and language/verbal skills.

Minzenberg et al. (41) using clinical and pharmacological in vitro indicators reported a significant association of high anticholinergic load with worse declarative memory performance. Specifically, the study found that an increase in anticholinergic load from 0.5 to $4 \mathrm{mg} /$ day equivalent pharmacological benztropine can lead to a reduction of up to $1.7 \mathrm{SD}$ of the California verbal learning test performance. In 
TABLE 1 | Qualitative analysis with the main characteristics of studies related to the influence of anticholinergic tapering on cognition in schizophrenia.

\begin{tabular}{|c|c|c|c|c|c|c|c|c|c|c|}
\hline $\begin{array}{l}\text { References, } \\
\text { study design }\end{array}$ & Country & Setting & Population & Age (years) & $\%$ Female & Study groups & $\begin{array}{l}\text { Cofounders } \\
\text { studied }\end{array}$ & $\begin{array}{l}\text { Intervention } \\
\text { assessment } \\
\text { method }\end{array}$ & $\begin{array}{l}\text { Outcome } \\
\text { assessment } \\
\text { method }\end{array}$ & Summary of findings \\
\hline $\begin{array}{l}\text { Drimer et al. (49), } \\
\text { prospective cohort } \\
\text { study }\end{array}$ & Israel & $\begin{array}{l}\text { Inpatients from } \\
\text { Abarbanel Mental } \\
\text { Health Center, } \\
\text { Bat-Yam, Israel }\end{array}$ & $n=27$ & $\begin{array}{l}\text { Mean age } 65.7 \\
\text { years }\end{array}$ & $51.85 \%$ female & No control group & $\mathrm{N} / \mathrm{A}$ & $\begin{array}{l}\text { Anticholinergic } \\
\text { tapering }\end{array}$ & ADAS-Cog & $\begin{array}{l}\text { Biperiden tapering } \\
\text { showed significant } \\
\text { improvement in } \\
\text { ideational praxis, } \\
\text { orientation, and overall } \\
\text { score of ADAS-Cog. } \\
\text { Improvement } \\
\text { correlated with } \\
\text { previous dose of } \\
\text { biperiden. No adverse } \\
\text { events/extrapyramidal } \\
\text { symptoms }\end{array}$ \\
\hline $\begin{array}{l}\text { Desmarais et al. } \\
\text { (48), prospective } \\
\text { cohort study }\end{array}$ & Canada & $\begin{array}{l}\text { Outpatients from } \\
\text { Schizophrenia } \\
\text { Tertiary Services } \\
\text { outpatient clinic of } \\
\text { the McGill } \\
\text { University Health } \\
\text { Center }\end{array}$ & $\begin{array}{l}n=20 \text { with } \\
\text { schizophrenia or } \\
\text { schizoaffective } \\
\text { disorder }\end{array}$ & $52.7 \pm 7.8$ years & $42.86 \%$ female & No control group & $\begin{array}{l}\text { Gender, Age, } \\
\text { Education level, } \\
\text { Age at onset of } \\
\text { illness, Length of } \\
\text { illness, Presence } \\
\text { of parkinsonism, } \\
\text { dystonia, or } \\
\text { tardive dyskinesia }\end{array}$ & $\begin{array}{l}\text { Anticholinergic } \\
\text { tapering }\end{array}$ & $\begin{array}{l}\text { ESRS, BACS or } \\
\text { BECS (French } \\
\text { version) depending } \\
\text { on patients' } \\
\text { language, PANSS, } \\
\text { CGI-S CGI-I }\end{array}$ & $\begin{array}{l}\text { Significant } \\
\text { improvement on BACS } \\
\text { z score at weeks } 6,8, \\
\text { and } 12 \text {, especially on } \\
\text { motor and } \\
\text { symbol-coding tasks } \\
\text { after anticholinergic } \\
\text { tapering. No significant } \\
\text { effects on the PANSS, } \\
\text { CGI-S, and CGI-I }\end{array}$ \\
\hline $\begin{array}{l}\text { Ogino et al. (50), } \\
\text { prospective cohort } \\
\text { study }\end{array}$ & Japan & $\begin{array}{l}\text { Inpatients and } \\
\text { Outpatients from } \\
\text { St. Marianna } \\
\text { University School } \\
\text { of Medicine } \\
\text { Hospital and Ofuji } \\
\text { Hospital }\end{array}$ & $\begin{array}{l}n=24 \text { biperiden } \\
\text { tapering group } \\
\text { schizophrenia } \\
\text { patients, } n=10 \\
\text { control group }\end{array}$ & $\begin{array}{l}\text { Biperiden tapering } \\
\text { group } \\
\text { schizophrenia } \\
\text { patients: } 35.7 \pm \\
12 \text { years } \\
\text { Control group: } \\
43.5 \pm 8.7 \text { years }\end{array}$ & $\begin{array}{l}\text { Biperiden tapering } \\
\text { group } \\
\text { schizophrenia } \\
\text { patients: } 45.83 \% \\
\text { female } \\
\text { Control group: } \\
50 \% \text { female }\end{array}$ & $\begin{array}{l}\text { Biperiden tapering } \\
\text { group, control } \\
\text { group } \\
\text { schizophrenia } \\
\text { patients }\end{array}$ & $\begin{array}{l}\text { Gender, age, } \\
\text { education, age at } \\
\text { onset of illness, } \\
\text { duration of } \\
\text { untreated } \\
\text { psychosis, } \\
\text { duration of } \\
\text { biperiden } \\
\text { administration/or } \\
\text { mean daily dose of } \\
\text { biperiden, } \\
\text { antipsychotic } \\
\text { drugs, or } \\
\text { benzodiazepines }\end{array}$ & $\begin{array}{l}\text { Biperiden } \\
\text { tapering }\end{array}$ & $\begin{array}{l}\text { BACS-J, SQLS-J, } \\
\text { PANSS }\end{array}$ & $\begin{array}{l}\text { Significant } \\
\text { improvements in } \\
\text { attention, processing } \\
\text { speed, and composite } \\
\text { score (BACS-J), in } \\
\text { psychosocial condition } \\
\text { score (SQLS-J) and } \\
\text { general } \\
\text { psychopathology score } \\
\text { (PANSS) after biperiden } \\
\text { tapering }\end{array}$ \\
\hline
\end{tabular}

ADAS-cog, Alzheimer's disease assessment scale-cognitive; BACS, Brief assessment of cognition in schizophrenia; BACS-J, Brief assessment of cognition in schizophrenia-Japan, BECS, brève evaluation de la cognition en schizophrénie; CGIII, Clinical global impression-improvement scales; CGI-S, Clinical global impression-severity scales; ESRS, Extrapyramidal symptom rating scale; PANSS, Positive and negative syndrome scale; SQLS-J, Schizophrenia quality of life scale-Japan. 
TABLE 2 | Qualitative analysis with the main characteristics of studies related to the influence of medication anticholinergic burden on cognition in schizophrenia.

\begin{tabular}{|c|c|c|c|c|c|c|c|c|c|c|}
\hline $\begin{array}{l}\text { References, } \\
\text { study design }\end{array}$ & Country & Setting & Population & Age (years) & $\%$ Female & Study groups & $\begin{array}{l}\text { Cofounders } \\
\text { studied }\end{array}$ & $\begin{array}{l}\text { Intervention } \\
\text { assessment } \\
\text { method }\end{array}$ & $\begin{array}{l}\text { Outcome } \\
\text { assessment method }\end{array}$ & Summary of findings \\
\hline $\begin{array}{l}\text { Kim et al. (40), } \\
\text { cross sectional } \\
\text { study }\end{array}$ & South Korea & $\begin{array}{l}\text { Inpatients from a } \\
\text { university hospital } \\
\text { and } 2 \text { mental } \\
\text { hospitals }\end{array}$ & $\begin{array}{l}n=60, \text { Low ADS } \\
n=31 \\
\text { High ADS } n=29\end{array}$ & $\begin{array}{l}\text { Low ADS: } 35.61 \\
\pm 7.26 \text { years } \\
\text { High ADS: } 42.59 \\
\pm 10.66 \text { years }\end{array}$ & $\begin{array}{l}\text { Low ADS: } \\
\text { 25.81\% female, } \\
\text { High ADS: } \\
31.03 \% \text { female }\end{array}$ & $\begin{array}{l}A D S \geq 3 \text { group } \\
\text { and } A D S<3\end{array}$ & $\begin{array}{l}\text { Gender, age, } \\
\text { depression, } \\
\text { education }\end{array}$ & ADS & $\begin{array}{l}\text { MCCB for cognitive } \\
\text { functions/UPSA for } \\
\text { daily living functions }\end{array}$ & $\begin{array}{l}\text { Statistically negative } \\
\text { association between } \\
\text { high anticholinergic } \\
\text { burden and poorer } \\
\text { cognitive (composite } \\
\text { MCCB score) and daily } \\
\text { living functions (total } \\
\text { UPSA score) }\end{array}$ \\
\hline $\begin{array}{l}\text { Ang et al. (38), } \\
\text { cross sectional } \\
\text { study }\end{array}$ & China & $\begin{array}{l}\text { Outpatients/ } \\
\text { inpatients from the } \\
\text { Institute of Mental } \\
\text { Health, Singapore, } \\
\text { community care } \\
\text { centers and } \\
\text { rehabilitation } \\
\text { centers in } \\
\text { Singapore }\end{array}$ & $n=705$ & $\begin{array}{l}39.18 \pm 9.71 \\
\text { years }\end{array}$ & $47.2 \%$ female & No control group & $\begin{array}{l}\text { Duration and } \\
\text { severity of illness, } \\
\text { antipsychotic } \\
\text { dose, smoking } \\
\text { status, age, } \\
\text { gender }\end{array}$ & ABS and ADS & $\begin{array}{l}\text { Neuropsychological } \\
\text { battery (JLO, WASI-II, } \\
\text { CPT-IP, BACS) }\end{array}$ & $\begin{array}{l}\text { Anticholinergic burden } \\
\text { was negatively } \\
\text { correlated with } \\
\text { cognitive performance } \\
\text { in global cognition } \\
\text { (executive function, } \\
\text { memory/fluidity, } \\
\text { processing speed) but } \\
\text { due to the small size of } \\
\text { the association, the } \\
\text { clinical significance is } \\
\text { doubtful }\end{array}$ \\
\hline $\begin{array}{l}\text { Eum et al. (39), } \\
\text { cross sectional } \\
\text { study }\end{array}$ & United States & $\begin{array}{l}\text { From the bipolar- } \\
\text { schizophrenia } \\
\text { network on } \\
\text { intermediate } \\
\text { phenotypes } \\
\text { (B-SNIP) } \\
\text { consortium }\end{array}$ & $\begin{array}{l}n=206 \text { with } \\
\text { schizophrenia } \\
n=131 \text { with } \\
\text { schizoaffective } \\
\text { disorder } \\
n=146 \text { with } \\
\text { psychotic } \\
\text { bipolar disorder }\end{array}$ & $\begin{array}{l}\text { With } \\
\text { schizophrenia: } \\
36.37 \pm 13.21 \\
\text { years, with } \\
\text { schizoaffective } \\
\text { disorder: } 36.83 \pm \\
11.84 \text { years, with } \\
\text { psychotic bipolar } \\
\text { disorder: } 35.08 \pm \\
12.20 \text { years }\end{array}$ & $\begin{array}{l}\text { With } \\
\text { schizophrenia: } \\
33.5 \% \text { female, } \\
\text { with } \\
\text { schizoaffective } \\
\text { disorder: } 59.5 \% \\
\text { female, with } \\
\text { psychotic bipolar } \\
\text { disorder: } 62.3 \% \\
\text { female }\end{array}$ & No control group & $\begin{array}{l}\text { Age, gender, } \\
\text { symptom severity } \\
\text { (PANSS total } \\
\text { score), } \\
\text { antipsychotic } \\
\text { burden (CPZeq), } \\
\text { education, race }\end{array}$ & ADS & BACS & $\begin{array}{l}\text { ADS scores } \geq 4 \text { had } \\
\text { lower composite BACS } \\
\text { scores than those with } \\
A D S<4 \text { ( } p=0.004) \text {. } \\
\text { Verbal memory showed } \\
\text { statistically worse } \\
\text { performance in the high } \\
\text { anticholinergic load } \\
\text { group }\end{array}$ \\
\hline $\begin{array}{l}\text { Rehse et al. (19), } \\
\text { retrospective } \\
\text { cohort study }\end{array}$ & Germany & $\begin{array}{l}\text { Psychiatric } \\
\text { outpatient unit for } \\
\text { cognitive training } \\
\text { of the psychiatric } \\
\text { department at the } \\
\text { Heidelberg } \\
\text { University } \\
\text { Hospital, Germany }\end{array}$ & $\begin{array}{l}n=104 \text { with } \\
\text { schizophrenia }\end{array}$ & $28.2 \pm 8.6$ years & $59.5 \%$ female & $\begin{array}{l}\text { Group A-ADD } \\
\text { receivers, Group } \\
\text { B-ADD + CDD } \\
\text { receivers }\end{array}$ & $\begin{array}{l}\text { Age, gender, } \\
\text { education level, } \\
\text { time since onset } \\
\text { of illness }\end{array}$ & $\begin{array}{l}\text { ADD was } \\
\text { converted into } \\
\text { RIS-Eq/CPZ-Eq } \\
\text { CDD was } \\
\text { converted } \\
\text { into BZT-Eq }\end{array}$ & MCCB & $\begin{array}{l}\text { Significant negatively } \\
\text { correlation of ADD and } \\
\text { tasks of information } \\
\text { processing speed and } \\
\text { verbal memory. No } \\
\text { statistically significant } \\
\text { correlation of CDD and } \\
\text { cognitive performance }\end{array}$ \\
\hline
\end{tabular}


TABLE 2 | Continued

\begin{tabular}{|c|c|c|c|c|c|c|c|c|c|c|}
\hline $\begin{array}{l}\text { References, } \\
\text { study design }\end{array}$ & Country & Setting & Population & Age (years) & $\%$ Female & Study groups & $\begin{array}{l}\text { Cofounders } \\
\text { studied }\end{array}$ & $\begin{array}{l}\text { Intervention } \\
\text { assessment } \\
\text { method }\end{array}$ & $\begin{array}{l}\text { Outcome } \\
\text { assessment method }\end{array}$ & Summary of findings \\
\hline $\begin{array}{l}\text { Minzenberg et al. } \\
\text { (41), cross } \\
\text { sectional study }\end{array}$ & United States & $\begin{array}{ll}\text { Outpatients from } & r \\
\text { San Francisco VA } & s \\
\text { Medical Center } & s \\
\text { and surrounding } & c \\
\text { community } & r\end{array}$ & $\begin{array}{l}n=106 \text { with } \\
\text { schizophrenia or } \\
\text { schizoaffective } \\
\text { disorder } \\
n=50 \\
\text { control group }\end{array}$ & $\begin{array}{l}\text { Patients: } 39.9 \pm \\
11.3 \text { years } \\
\text { Control group: } \\
39.4 \pm 12.6\end{array}$ & $\begin{array}{l}\text { Patients: } 4 \% \\
\text { female } \\
\text { Control group: } \\
42 \% \text { female }\end{array}$ & $\begin{array}{l}\text { patients, healthy } \\
\text { subjects }\end{array}$ & $\begin{array}{l}\text { Age, Parental } \\
\text { education, } \\
\text { Parental } \\
\text { occupation level, } \\
\text { symptoms } \\
\text { severity, global } \\
\text { function }\end{array}$ & $\begin{array}{l}\text { Pharmacological } \\
\text { index from } \\
\text { published studies } \\
\text { Clinical index from } \\
\text { clinician ratings of } \\
\text { anticholinergic } \\
\text { medication } \\
\text { adverse effects }\end{array}$ & $\begin{array}{l}\text { Neuropsychological } \\
\text { battery (WAIS-R, } \\
\text { TMT-A/B, digit } \\
\text { span/visual span from } \\
\text { WMS-R, wisconsin } \\
\text { card sorting test, } \\
\text { stroop color and word } \\
\text { test, victoria version, } \\
\text { rey-osterrieth complex } \\
\text { figure design, california } \\
\text { verbal learning test, } \\
\text { facial memory from test } \\
\text { of memory and } \\
\text { learning, serial } \\
\text { visuospatial learning } \\
\text { test, controlled oral } \\
\text { word association test, } \\
\text { ruff figural fluency test, } \\
\text { finger tapping test) } \\
\text { extended version of } \\
\text { PANSS }\end{array}$ & $\begin{array}{l}\text { Anticholinergic load } \\
\text { associated with lower } \\
\text { scores on attention, } \\
\text { declarative memory, } \\
\text { and verbal memory } \\
\text { t }\end{array}$ \\
\hline $\begin{array}{l}\text { Tsoutsoulas et al. } \\
\text { (45), cross } \\
\text { sectional study }\end{array}$ & Canada & $\begin{array}{l}\text { Community- } \\
\text { Dwelling } \\
\text { patients }\end{array}$ & $n=60$ & $\geq 50$ years old & & No control group & $\mathrm{N} / \mathrm{A}$ & ACB & $\begin{array}{l}\text { CANTAB Alzheimer's } \\
\text { Dementia Battery for } \\
\text { cognitive deficits and } \\
\text { Repeatable Battery for } \\
\text { neuropsycological } \\
\text { status measures }\end{array}$ & $\begin{array}{l}\text { Anticholinergic burden } \\
\text { had significant negative } \\
\text { impact in spatial } \\
\text { working, short-term } \\
\text { memory, visuospatial } \\
\text { ability, and a negative } \\
\text { trend level of } \\
\text { correlation with learning } \\
\text { performance. No } \\
\text { adverse effects on } \\
\text { attention, executive } \\
\text { function, language, or } \\
\text { reaction time }\end{array}$ \\
\hline $\begin{array}{l}\text { Joshi et al. (47), } \\
\text { cross sectional } \\
\text { study }\end{array}$ & United States & $\begin{array}{l}\text { Outpatients from } \\
\text { five U.S. } \\
\text { universities -part of } \\
\text { the Consortium on } \\
\text { the Genetics of } \\
\text { Schizophrenia-2 }\end{array}$ & $n=1,120$ & $\begin{array}{l}\text { Mean age: } 46 \\
\text { years old }\end{array}$ & $\begin{array}{l}\text { Patients: } 30 \% \\
\text { female }\end{array}$ & $\begin{array}{l}\mathrm{ACB}=0, \\
\mathrm{ACB}=1 \text { or } 2 \\
\text { (low), } \mathrm{ACB}=3 \\
\text { or } 4 \text { (moderate), } \\
\mathrm{ACB}=5 \text { or } 6 \\
\text { (high), } \mathrm{ACB}>6 \\
\text { (very high) groups }\end{array}$ & \multicolumn{2}{|c|}{$\begin{array}{l}\text { Age, Demographic ACB } \\
\text { characteristic, } \\
\text { illeness severity, } \\
\text { Antipsychotic } \\
\text { burden (CPZeq), } \\
\text { clinical symptoms }\end{array}$} & $\begin{array}{l}\text { Penn Computerized } \\
\text { Neurocognitive Battery } \\
\text { (PCNB) }\end{array}$ & $\begin{array}{l}\text { Anticholinergic burden } \\
\text { had significant negative } \\
\text { impact on cognitive } \\
\text { performance across all } \\
\text { cognitive domains }\end{array}$ \\
\hline
\end{tabular}




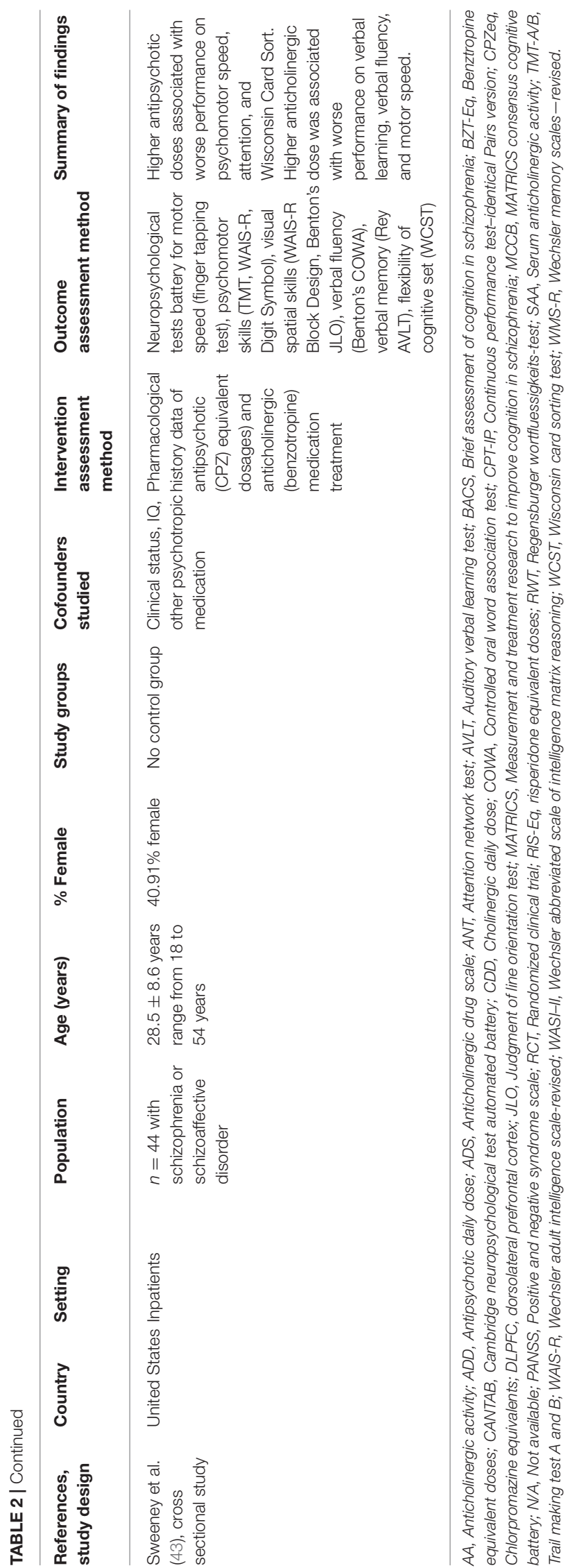

addition, Sweeney et al. (43) found that higher anticholinergic doses were also correlated with worse performance on verbal fluency (Verbal Fluency test) and verbal memory tasks (Rey AVLT). Similarly, memory/fluency performance although the small effect size was also reported to have a significant negative association with anticholinergic load evaluated with $A B C$ and ADS scales in Ang's et al. (38). Furthermore, Eum's et al. crosssectional study (39) reported statistically worse performance in the high anticholinergic load group (ADS $\geq 4$ ) compared to the low anticholinergic group ADS $<4$ in verbal memory task with a difference of 0.55 SDs. Significant are also the findings of the Vinogradov et al. study (53) in which high SAA was significantly associated with worse performance in the MCCB domain of verbal learning and memory $(r=-0.29, p<0.04)$, with which after regression analysis, SAA showed a common variation of $7 \%$, after controlling for the impact of age, IQ, and severity of symptoms. In addition, the cognitive effects of anticholinergic load, evaluated according to a different anticholinergic scale (ACB scale), in older patients with schizophrenia ( $\geq 50$ years), bear a similarity to cognitive dysfunction that occur in the early stages of Alzheimer's disease. High anticholinergic load was correlated with short-term memory decline $(p=0.004)$ evaluated with Alzheimer's Dementia Battery and the Repeatable Battery for the Assessment of Neuropsychological Status. It is also worth noting the emergence of a correlation tendency between high anticholinergic load and poorer learning performance (45).

\section{Anticholinergic Burden and Retrieval}

As regards specifically the performance of memory recall or retrieval (free recall, cued recall, and recognition), which refers to the mental process of retrieval of the information/events that were previously encoded and stored in the brain, in total four studies found a significant negative association with high SAA.

Specifically, in Tune's et al. cross-sectional study (46) the performance of the free recall test was of statistical importance $(r=0.51, p<0.01)$ demonstrating a negative effect of the high anticholinergic load on recent or working memory. On the other hand, a cross sectional study (42) with also a small sample size $(n=17)$ of chronic patients, similar age range but serum anticholinergicity due more to neuroleptic treatment than to antiparkinsonian drugs, was only partly in line with Tune's et al. (46) on the correlation between anticholinergic load and recall memory impairment $(r=-0.54, p=0.01)$. As regards the recognition memory, which was evaluated with the MattisKovner inventory test, no association with the anticholinergic load $(r=0.28, p>0.10)$ was found. Recall performance also declined (List Recall=total free recall; $t=-2.16, p=0.037$ ) in the group with the high serum anticholinergic load in the study of Tracy et al. (44) in which after statistical analysis, this task showed common variance with serum anticholinergicity of $16 \%$ $(r=0.40)$. In addition, the Hitri et al. (52) found that during the period of anticholinergic treatment, in which there was an increase in SAA, there was a decrease in the number of recall words by $22 \%$ compared to the starting point. Short-term recall memory decreased by $31 \%$, but long-term memory did not show any statistically significant change due to the anticholinergic agents administered. 
TABLE 3 | Qualitative analysis with the main characteristics of studies related to the influence of SAA on cognition in schizophrenia.

\begin{tabular}{|c|c|c|c|c|c|c|c|c|c|c|}
\hline $\begin{array}{l}\text { References, } \\
\text { study design }\end{array}$ & Country & Setting & Population & Age (years) & $\%$ Female & Study groups & $\begin{array}{l}\text { Cofounders } \\
\text { studied }\end{array}$ & $\begin{array}{l}\text { Intervention } \\
\text { assessment } \\
\text { method }\end{array}$ & $\begin{array}{l}\text { Outcome } \\
\text { assessment method }\end{array}$ & Summary of findings \\
\hline $\begin{array}{l}\text { Vinogradov et al. } \\
\text { (53), Single blind } \\
\text { randomized } \\
\text { clinical trial }\end{array}$ & United States & $\begin{array}{l}\text { Outpatients from } \\
\text { community mental } \\
\text { health centers }\end{array}$ & $\begin{array}{l}n=55 \\
\text { auditory } \\
\text { training group } \\
n=25 ; \\
\text { control group } \\
n=24\end{array}$ & $\begin{array}{l}\text { Auditory training } \\
\text { group: } 41.44 \pm \\
11,06 \text { years } \\
\text { Control Group: } \\
46.38 \pm \\
8.97 \text { years }\end{array}$ & $\begin{array}{l}\text { Auditory training } \\
\text { group: } 32 \% \\
\text { female } \\
\text { Control group: } \\
28 \% \text { female }\end{array}$ & $\begin{array}{l}\text { Auditory training } \\
\text { group and control } \\
\text { group }\end{array}$ & $\begin{array}{l}\text { Age, gender, } \\
\text { education, and } \\
\text { symptom severity, } \\
\text { IQ }\end{array}$ & $\begin{array}{l}\text { SAA with } \\
\text { radioreceptor } \\
\text { assay }\end{array}$ & $\begin{array}{l}\text { Neurocognitive battery } \\
\text { based on MATRICS }\end{array}$ & $\begin{array}{l}\text { Higher SAA significantly } \\
\text { correlated with worse } \\
\text { verbal working } \\
\text { memory, verbal } \\
\text { learning-memory, and } \\
\text { global cognition } \\
\text { change after auditory } \\
\text { training }\end{array}$ \\
\hline $\begin{array}{l}\text { Tune et al. (46), } \\
\text { cross sectional } \\
\text { study }\end{array}$ & United States & Outpatients & $\begin{array}{l}n=24 \text { from } \\
20 \text { to } 58 \\
\text { years }\end{array}$ & Mean age 35.7 & $45.83 \%$ female & No control group & N/A & $\begin{array}{l}\text { SAA with } \\
\text { radioreceptor } \\
\text { assay of Creese } \\
\text { and Snyder for } \\
\text { neuroleptics and } \\
\text { radioreceptor } \\
\text { assay of Tune and } \\
\text { Coyle for } \\
\text { anticholinergics }\end{array}$ & $\begin{array}{l}\text { Free recall memory } \\
\text { test, WAIS, structured } \\
\text { interview by psychiatrist }\end{array}$ & $\begin{array}{l}\text { Significant correlation } \\
\text { between high SAA and } \\
\text { recall test performance }\end{array}$ \\
\hline $\begin{array}{l}\text { Tracy et al. (44), } \\
\text { cross sectional } \\
\text { study }\end{array}$ & United States & $\begin{array}{l}\text { Patients at } \\
\text { Norristown State } \\
\text { Hospital }\end{array}$ & $n=38$ & $39.7 \pm 10.2$ years & $32 \%$ female & $\begin{array}{l}\text { High/low } \\
\text { anticholinergic } \\
\text { group }\end{array}$ & $\begin{array}{l}\text { Gender, age, } \\
\text { education level, } \\
\text { Smoking, alcohol } \\
\text { duration of illness, } \\
\text { other neurological } \\
\text { diseases, } \\
\text { antipsychotic dose }\end{array}$ & $\begin{array}{l}\text { SAA with } \\
\text { radioreceptor } \\
\text { assay of Tune and } \\
\text { Coyle }\end{array}$ & $\begin{array}{l}\text { CPT-IP for selective } \\
\text { attention, Stroop Test } \\
\text { for "inhibitory" } \\
\text { executive control, Digit } \\
\text { Vigilance Test for } \\
\text { sustained attention, a } \\
\text { single verbal memory } \\
\text { task for automatic and } \\
\text { effortful memory, finger } \\
\text { tapping test for } \\
\text { psychomotor speed }\end{array}$ & $\begin{array}{l}\text { Significant correlation } \\
\text { between high SAA and } \\
\text { worse performance on } \\
\text { executive control and } \\
\text { effortful memory }\end{array}$ \\
\hline $\begin{array}{l}\text { Perlick et al. (42), } \\
\text { cross sectional } \\
\text { study }\end{array}$ & United States & $\begin{array}{l}\text { Inpatients from } \\
\text { psychiatric } \\
\text { hospital in New } \\
\text { York }\end{array}$ & $n=17$ & $\begin{array}{l}\text { Mean age } 33.4 \\
\text { years }\end{array}$ & $29.41 \%$ female & No control group & $\begin{array}{l}\text { IQ, Age, organic } \\
\text { impairment related } \\
\text { to mental capacity, } \\
\text { serum neuroleptic } \\
\text { load }\end{array}$ & $\begin{array}{l}\text { SAA with } \\
\text { radioreceptor } \\
\text { assay of Tune and } \\
\text { Coyle (31) for } \\
\text { anticholinergics } \\
\text { and Tune et al. (46) } \\
\text { for neuroleptics }\end{array}$ & $\begin{array}{l}\text { Neuropsychological } \\
\text { tests battery (WAIS-R, } \\
\text { Benton's revised visual } \\
\text { retention test, } \\
\text { mattis-kovner memory } \\
\text { inventory) brief } \\
\text { psychiatric rating scale }\end{array}$ & $\begin{array}{l}\text { Significant correlation } \\
\text { between high SAA and } \\
\text { verbal recall memory. } \\
\text { No association } \\
\text { between serum } \\
\text { anticholinergicity and } \\
\text { recognition memory }\end{array}$ \\
\hline
\end{tabular}


TABLE 3 | Continued

\begin{tabular}{|c|c|c|c|c|c|c|c|c|c|c|}
\hline $\begin{array}{l}\text { References, } \\
\text { study design }\end{array}$ & Country & Setting & Population & Age (years) & $\%$ Female & Study groups & $\begin{array}{l}\text { Cofounders } \\
\text { studied }\end{array}$ & $\begin{array}{l}\text { Intervention } \\
\text { assessment } \\
\text { method }\end{array}$ & $\begin{array}{l}\text { Outcome } \\
\text { assessment method }\end{array}$ & Summary of findings \\
\hline $\begin{array}{l}\text { Hitri et al. (52), } \\
\text { randomized } \\
\text { clinical trial }\end{array}$ & United States & $\begin{array}{l}\text { Inpatients from } \\
\text { Augusta Veterans } \\
\text { Administration } \\
\text { Hospital, Georgia }\end{array}$ & $n=15$ & $\begin{array}{l}\text { Range from } 28 \text { to } \\
60 \text { years }\end{array}$ & $0 \%$ female & $\begin{array}{l}\text { Group } \\
\text { benztropine; } \\
n=5 \text {, Group } \\
\text { trihexyphenidyl; } \\
n=5 \text {, Group } \\
\text { amantadine; } n=5\end{array}$ & N/A & $\begin{array}{l}\text { SAA with } \\
\text { radioreceptor } \\
\text { assay of Tune and } \\
\text { Coyle for } \\
\text { anticholinergics } \\
\text { and Creese and } \\
\text { Snyder for } \\
\text { neuroleptics }\end{array}$ & $\begin{array}{l}\text { Neuropsychological } \\
\text { tests battery for } \\
\text { attention, } \\
\text { concentration, memory } \\
\text { (digit span, selective } \\
\text { reminding memory task } \\
\text { by Buschke) }\end{array}$ & $\begin{array}{l}\text { Higher SAA correlated } \\
\text { with reduction of } \\
\text { short-term recall } \\
\text { performance but not } \\
\text { with long-term memory } \\
\text { function }\end{array}$ \\
\hline $\begin{array}{l}\text { Tracy et al. (51), } \\
\text { prospective cohort } \\
\text { study }\end{array}$ & United States & Inpatients & $n=22$ & $44.7 \pm 8.4$ years & $45.5 \%$ female & $\begin{array}{l}\text { Group clozapine; } \\
n=15, \text { Group } \\
\text { risperidone; } n=7\end{array}$ & $\begin{array}{l}\text { Age, Age at onset } \\
\text { of illness, other } \\
\text { neurological } \\
\text { disorders, } \\
\text { medication } \\
\text { possible to affect } \\
\text { cognition, race, } \\
\text { gender, education }\end{array}$ & $\begin{array}{l}\text { SAA with } \\
\text { radioreceptor } \\
\text { assay of Tune and } \\
\text { Coyle for } \\
\text { anticholinergics }\end{array}$ & $\begin{array}{l}\text { Neurocognitive test } \\
\text { battery }\end{array}$ & $\begin{array}{l}\text { Higher SAA of patients } \\
\text { treated with clozapine } \\
\text { than risperidone but no } \\
\text { differences on cognitive } \\
\text { functions }\end{array}$ \\
\hline
\end{tabular}

AA, Anticholinergic activity; ADD, Antipsychotic daily dose; ADS, Anticholinergic drug scale; ANT, Attention network test; AVLT, Auditory verbal learning test; BACS, Brief assessment of cognition in schizophrenia; BZT-Eq, Benztropine equivalent doses; CANTAB, Cambridge neuropsychological test automated battery; CDD, Cholinergic daily dose; COWA, Controlled oral word association test; CPT-IP, Continuous performance test-identical Pairs version; CPZeq, Chlorpromazine equivalents; DLPFC, dorsolateral prefrontal cortex; JLO, Judgment of line orientation test; MATRICS, Measurement and treatment research to improve cognition in schizophrenia; MCCB, MATRICS consensus cognitive battery; N/A, Not available; PANSS, Positive and negative syndrome scale; RCT, Randomized clinical trial; RIS-Eq, risperidone equivalent doses; RWT, Regensburger wortfluessigkeits-test; SAA, Serum anticholinergic activity; TMT-A/B, Trail making test $A$ and B; WAIS-R, Wechsler adult intelligence scale-revised; WASI-II, Wechsler abbreviated scale of intelligence matrix reasoning; WCST, Wisconsin card sorting test; WMS-R, Wechsler memory scales-revised. 


\section{Anticholinergic Burden and Executive Functions}

Executive function performance, which refers to a set of highorder cognitive processes such as working memory, inhibitory control, cognitive flexibility, planning, reasoning, and problem solving, was reported by five studies to be negatively affected with the increase of medication anticholinergic burden.

As regards working memory that is generally considered a key executive function domain, Vinogradov et al. (53) found that high SAA was significantly associated with worse performance in the MCCB domain of verbal working memory $(r=-0.41$, $p<0.04)$. Furthermore, high anticholinergic load assessed with ACB was also correlated with worse performance in spatial working memory $(p=0.04)$ in the study of Tsoutsoulas et al. (45).

In addition, in the Tracy et al. (44) patients treated only with a stable dose of single antipsychotic medication, higher SAA was associated with worse performance in Executive Control tests (Stroop interference index; $t=-2,6, p=0.015$ ), in which after statistical analysis, the task showed common variance with serum anticholinergicity of $19 \%(r=-0.45)$. Similarly, in the Ang et al. (38) executive function performance (Judgment of Line Orientation test and Matrix Reasoning test) was also of significant importance; however, the effect size of both ABS and ADS scales was small questioning its clinical significance. It is also worth noting that a trend of effect on executive function was also observed in the retrospective cohort study of Rehse et al. (19) with the increase in daily pharmacological doses of anticholinergic drugs (CDD) $(b=0.280, p<0.10)$ but is likely to be symptomatic as no significant results were observed under the increasing CDD load neither in the whole sample nor in the subgroups.

\section{Anticholinergic Burden and Other Cognitive Domains}

Furthermore, the domains of processing speed (including non-motor/cognitive activity or motor/physical activity), divided attention, and visual-spatial ability have been reported to be negatively associated with high medication anticholinergic burden.

Specifically, higher doses of anticholinergic benztropine were correlated with worse performance on motor speed (Finger Tapping, Digit Symbol) in Sweeney et al. (43) with clinically stable patients with schizophrenia, aged from 18 to 54 years old. On the other hand, in the study of Rehse et al. (19), CDD did not show significant influence in any cognitive field in the regression analysis of the entire sample; however, in the subgroup examination regarding antipsychotics' low or high anticholinergic binding profile, the increase in the anticholinergic load was associated with significantly lower data processing speed $(b=0.292, p<0.05)$, as opposed to treatment with antipsychotics without anticholinergic properties. With the application of locally weighted scatterplot smoothing tool to verify the relationship of cognitive function with the increase in anticholinergic daily dose, although the tendency for reduced speed of information processing was confirmed by increasing CDD, a paradox was observed, since the group of the highest anticholinergic dose $(\mathrm{BZT}-\mathrm{Eq}>20)$ exhibited a higher rate of information processing than the group of $0<$ BZT-Eq $<15$. Nevertheless, this could be attributed either to insufficient data on anticholinergic equivalent doses for certain drugs or to the specific pharmacological properties of clozapine taken by all patients as the major antipsychotic drug, which could compensate for the adverse anticholinergic effects. Poorer processing speed/vigilance performance was also associated with the high anticholinergic burden in the study of Ang et al. (38), however the effect size was small for both anticholinergic classification scales (ABS and ADS).

Furthermore, the domain of divided attention was reported by Minzenberg et al. (41) to be negatively correlated with high anticholinergic load evaluated with pharmacological index from published studies and clinical index from clinician ratings of anticholinergic medication adverse effects (45). In addition, high ACB scores were correlated with worse visuospatial ability performance $(p=0.02)$ evaluated with Alzheimer's Dementia Battery and the Repeatable Battery for the Assessment of Neuropsychological Status in Tsoutsoulas et al. (45).

\section{Quality Assessment}

Table 4 presents the results of the quality assessment based on the Newcastle-Ottawa scale for cohort studies. The range of methodological quality score was between 3 and 9. Four articles $(19,48,50,51)$ had a medium quality to high quality and the one (49) had a low methodological quality. Based on the Newcastle-Ottawa quality assessment scale adapted for cross sectional studies, the range of methodological quality score of the included studies was between 6 and 9 (Table 5). Lastly, our quality assessment for clinical trials, presented in Table 6, shows that two studies $(52,53)$ range in a score of between 3 and 3.5, indicating low methodological quality.

\section{DISCUSSION}

To our knowledge, this is the first systematic review to examine the effect of cumulative anticholinergic burden on neurocognitive performance in patients with schizophrenia. Summing up the results of the studies included, it is largely concluded that medication with an increased anticholinergic load was observed in most studies as likely to affect the cognitive and daily living functions of patients with schizophrenia. Notably, the affected cognitive domains despite the abnormal cholinergic neurotransmission observed in schizophrenia are in line with our knowledge of the effect of cholinergic modulation on cognitive processes in healthy individuals (54). In the majority of studies, the increase in anticholinergic load was associated with impaired global cognition or decreased composite scores of several neurocognitive batteries as well as with decline of individual cognitive domains such as learning and memory, processing speed, executive function, and attention, which constitute key cognitive deficits of the disease (55). Although the studies with the highest methodological quality score $(38,39,50)$ indicate a positive association of the anticholinergic load with cognitive deficiencies, the findings of one of the largest study of this systematic review (38) are characterized by a small effect size and are of dubious clinical importance. The various cognitive domains reported in the studies included may be due to the heterogeneity of the diagnostic evaluation tools used to assess cognitive functions. 
TABLE 4 | Quality assessment of cohort studies (34).

\begin{tabular}{|c|c|c|c|c|c|c|c|c|c|}
\hline \multirow[t]{2}{*}{ References } & \multicolumn{4}{|c|}{ Selection } & \multirow{2}{*}{$\begin{array}{c}\text { Comparability } \\
(1) \\
\text { Comparability of } \\
\text { cohorts on the } \\
\text { basis of the } \\
\text { design or } \\
\text { analysis }^{\mathrm{a}}\end{array}$} & \multicolumn{3}{|c|}{ Outcome } & \multirow{2}{*}{$\begin{array}{l}\text { Total quality } \\
\text { score }\end{array}$} \\
\hline & $\begin{array}{c}\text { (1) } \\
\text { Representativeness } \\
\text { of the exposed } \\
\text { cohort }\end{array}$ & $\begin{array}{c}\text { (2) } \\
\text { Selection of the } \\
\text { non-exposed } \\
\text { cohort }\end{array}$ & $\begin{array}{c}\text { (3) } \\
\text { Ascertainment } \\
\text { of exposure }\end{array}$ & $\begin{array}{c}\text { (4) } \\
\text { Demonstration } \\
\text { that outcome of } \\
\text { interest was not } \\
\text { present at start } \\
\text { of study }\end{array}$ & & $\begin{array}{c}\text { (1) } \\
\text { Assessment of } \\
\text { outcome }\end{array}$ & $\begin{array}{c}\text { (2) } \\
\text { Was follow-up } \\
\text { long enough for } \\
\text { outcomes to } \\
\text { occur? }\end{array}$ & $\begin{array}{l}\text { (3) Adequacy of } \\
\text { follow up of } \\
\text { cohorts }\end{array}$ & \\
\hline Rehse et al. (19) & $\star$ & $\star$ & $\star$ & & $\star \star$ & $\star$ & $\star$ & * & 8 \\
\hline $\begin{array}{l}\text { Desmarais et al. } \\
\text { (48) }\end{array}$ & 夫 & & $\star$ & * & & * & * & * & 6 \\
\hline Ogino et al. (50) & $\star$ & $\star$ & $\star$ & $\star$ & $\star \star$ & * & * & * & 9 \\
\hline Drimer et al. (49) & & & $\star$ & & & & $\star$ & $\star$ & 3 \\
\hline Tracy et al. (51) & $\star$ & $\star$ & $\star$ & $\star$ & $\star \star$ & * & & $\star$ & 8 \\
\hline
\end{tabular}

${ }^{a}$ A maximum of 2 stars can be awarded for this item. A study controlling for age receives one star, and a study controlling for other major risk factors receives an additional star.

TABLE 5 | Quality assessment of cross-sectional studies (35).

\begin{tabular}{|c|c|c|c|c|c|c|c|c|}
\hline \multirow[t]{2}{*}{ References } & \multicolumn{4}{|c|}{ Selection $^{a}$} & \multirow{2}{*}{$\begin{array}{c}\text { Comparability } \\
(1) \\
\text { Comparability of subjects } \\
\text { on the basis of the } \\
\text { design or analysis }\end{array}$} & \multicolumn{2}{|c|}{ Outcome $^{c}$} & \multirow{2}{*}{$\begin{array}{l}\text { Total quality } \\
\text { score }\end{array}$} \\
\hline & $\begin{array}{l}\text { (1) } \\
\text { Representativeness } \\
\text { of the sample }\end{array}$ & $\begin{array}{c}\text { (2) } \\
\text { Sample size }\end{array}$ & $\begin{array}{c}\text { (3) } \\
\text { Non-responders }\end{array}$ & $\begin{array}{c}\text { (4) } \\
\text { Ascertainment } \\
\text { of the exposure }\end{array}$ & & $\begin{array}{c}\text { (1) } \\
\text { Assessment of } \\
\text { the outcome }\end{array}$ & $\begin{array}{c}(2) \\
\text { Statistical test }\end{array}$ & \\
\hline
\end{tabular}

Joshi et al. (47)

Kim et al. (40)

Ang et al. (38)

Eum et al. (39)

Minzenberg et al. (41)

Tune et al. (46)

Tracy et al. (44)

Perlick et al. (42)

Tsoutsoulas et al. (45)

Sweeney et al. (43)

${ }^{a} A$ maximum of 5 stars can be awarded for the selection.

${ }^{b} A$ maximum of 2 stars can be awarded for the comparability.

${ }^{\circ}$ A maximum of 3 stars can be awarded for the outcome.

$\begin{array}{ll}\star & \star \star \\ \star \star & \star \star \\ \star \star & \star \star \\ \star \star & \star \star \\ \star & \star \\ \star \star & \star \\ \star \star & \star \star \\ \star & \star \\ \star & \star \\ \star & \star\end{array}$

$\begin{array}{lll}\star \star & \star & 8 \\ \star \star & \star & 8 \\ \star \star & \star & 9 \\ \star \star & \star & 9 \\ \star \star & \star & 7 \\ \star \star & & 6 \\ \star \star & \star & 8 \\ \star \star & \star & 8 \\ \star \star & \star & 7 \\ \star \star & \star & 6\end{array}$




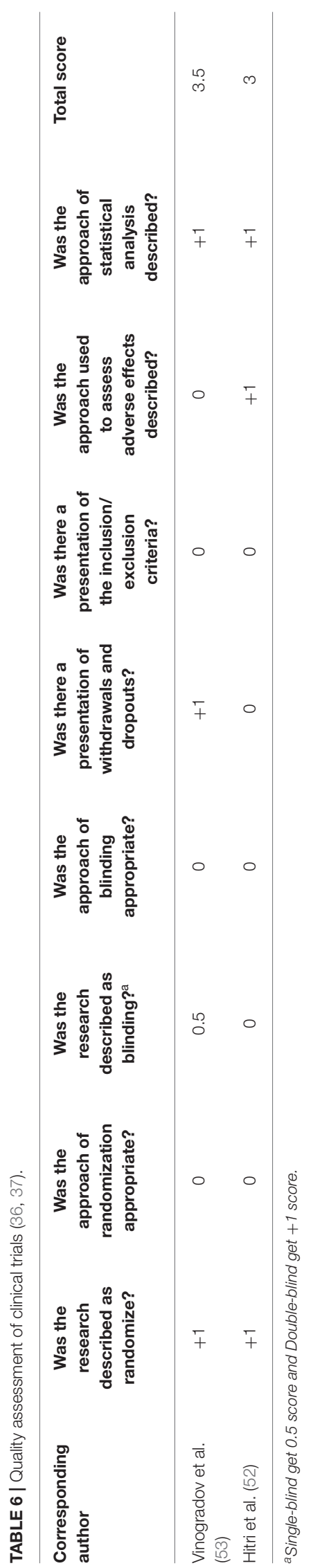

Heretofore several systematic reviews have compared typical and atypical antipsychotic drugs in terms of their influence on the cognitive functions of patients with schizophrenia, with some studies suggesting that the use of atypical antipsychotics improves cognitive functions (56), whereas findings from other studies appear to support that both first and second generation antipsychotics have the same effect on cognitive functions (57). In general, cognitive impairment due to medication is claimed to be related to the affinity of drugs with receptors, and cognitive dysfunction is observed with drugs that are muscarinic cholinergic, a2 adrenergic, 5-HT2A/2C serotonergic, and D1/D2 dopaminergic blockers or agonists of 5-HT1A serotonin receptors. As far as the affinity of second-generation antipsychotics with anticholinergic receptors is concerned, this seems to vary (56). It is worth noting that the recent metaanalysis of Tani et al. (58), which included two randomized clinical trials that investigated antipsychotic dose reduction, found that $50 \%$ of SGAs dose reduction significantly improved neurocognitive performance. Thus, this indicates that atypical antipsychotics can also have harmful effects on neurocognitive function. These findings could also be attributed to the cumulative anticholinergic burden of antipsychotics as well as their antidopaminergic properties in the prefrontal cortex which have been associated with cognitive impairment (59). Therefore, in addition to the dose-dependent effects of antipsychotics on neurocognitive performance, the evaluation of antipsychotic therapy based on its anticholinergic profile as performed in our systematic review may provide a useful different approach for research and clinical purposes.

It is also worth discussing the findings of studies on antipsychotic clozapine $(19,51)$, which although considered a high potency anticholinergic agent, does not seem to affect cognitive performance to the same extent as other drugs with similar anticholinergic properties. These findings are in line with other studies which indicate the improvement of cognitive functions in patients with schizophrenia who were treated with antipsychotic clozapine $(60,61)$. The specificities regarding the action of clozapine are likely attributed to the distinct pharmacological characteristics related to its affinity with different receptors and thus the action on different neurotransmission systems (62). Furthermore, recent studies found that the ratio of clozapine to its active metabolite norclozapine (N-Desmethylclozapine) in plasma is associated with patients' cognitive performance. Clozapine has different pharmacodynamic properties and affinity to cholinergic, dopaminergic, and serotonergic receptors compared to its active metabolite and literature supports that lower clozapine/norclozapine ratios in clinically stable patients are associated with better cognitive outcome $(63,64)$. The metabolism of clozapine to norclozapine is induced by P450 enzymatic system and thus adjunctive treatments, caffeine consumption, smoking, and other factors such as age, race, and gender may alter this ratio between individuals $(65,66)$. Therefore, the consideration of all these confounding factors related to the complex pharmacological properties of clozapine is necessary for the interpretation of the findings of these studies. 
In studies that assessed the impact of anticholinergic withdrawal on cognitive performance using different cognitive evaluation tools, successful discontinuation of anticholinergic medication by most patients was achieved, with a significant improvement in cognitive functions and without worsening of extrapyramidal symptoms or psychopathology compared to the baseline. Thus, this implies that anticholinergics do not cause long-term effects on cognition, which can even be reversed after withdrawal, and that long-term use may not be necessary in most patients receiving antipsychotics. Findings are also in agreement and support existing clinical guidelines, which do not recommend the prophylactic use of anticholinergic drugs, recommending anticholinergics co-administration only in the early stages of treatment if necessary and not for long-term use $(67,68)$. In the other hand, literature shows that despite the indications in clinical guidelines, the practice of administering anticholinergic therapy in combination with antipsychotics as well as antipsychotic polypharmacy varies across countries (69-73), with global rates of about 15\% (74) and 20\% (75) respectively. Furthermore, antipsychotic polypharmacy appears to be associated with cognitive impairment of schizophrenia patients, which could be due to the cumulative anticholinergic properties of drugs co-administered (74).

The systematic review underlines the urgent need for additional prospective studies (e.g., cohort studies, clinical trials) that will examine the longitudinal exposure in anticholinergic agents, with a more representative sample and longer monitoring duration to eliminate as many systemic errors and biases as possible and to draw more universal conclusions. More research is also certainly needed from a neurological point of view to explain and clarify the involvement of cholinergic neurotransmission and the general neurochemical mechanisms in the cognitive impairment of people with schizophrenia, since there are several hypotheses about various pathophysiological mechanisms that involve different neurochemical pathways and neurotransmitter systems including the role of muscarinic and nicotinic acetylcholine receptors (1,76-78). Although several antipsychotics have been developed over the past decade to treat schizophrenia, cognitive rehabilitation drugs have not yet been approved by the FDA; therefore, cognitive deficits observed in the disease remain a huge scourge on the lives of millions of people around the world $(79,80)$. Not surprisingly, according to the findings of this systematic review, cholinesterase inhibitors have been proposed in several clinical studies as an additional therapy to standard antipsychotic treatments, to address cognitive decline in patients with schizophrenia. There are different cholinesterase inhibitor drugs with varying affinity either for acetyl cholinesterase (AChE) or butyryl cholinesterase (BChE) enzyme that act by blocking the cholinesterase enzyme from metabolizing $\mathrm{ACh}$, leading to increased availability of ACh in neuron synapses (81). According to a previous systematic review, acetylcholinesterase inhibitor in combination with antipsychotic drug showed medium-sized improvements regarding the cognitive functions, particularly in the domains of attention, visual memory, verbal memory and language, and executive functioning (82). However, there is an urgent need for larger, well-designed randomized clinical trials for stronger evidence. Different approaches suggest in addition to memory enhancement drugs and cognitive training programs, which require more extensive study (83). Vinogradov et al. (53) reported a negative association between anticholinergic load and patients' response in the auditory training "based on neuroplasticity" programme, which has been supported by several studies as a promising approach to treating cognitive disorders in schizophrenia; therefore patients' medication history should be taken into account before assessing the effectiveness of the method $(84,85)$. Moreover, reassessment of the treatment already administered is important, since as we add to this review, it can affect cognitive functions, which may already be impaired due to the progressive degenerative nature of the disease.

As far as clinical practice is concerned, it is important for clinicians and other health professionals to assess the cumulative effects of anticholinergic drugs on cognition. Specifically, neurocognitive deficits can even explain about $20-60 \%$ of the variation in functional performance observed among patients with schizophrenia $(24,55,86-88)$. A systematic review and meta-analysis of 50 studies highlights that the average percentage of patients with schizophrenia with clinical and social recovery characteristics was only $13.5 \%$ (89), thus demonstrating the need to allocate more resources to develop new research strategies for the treatment of this disease. Literature reports that the cognitive domains which have been negatively affected by anticholinergic load in most studies of this systematic review, directly affect the independence, social inclusion, and occupational activities of patients. Specifically, according to a study, global cognition is more closely related to the disability of the disease than individual neurocognitive domains. However, deficits in the domains of executive function and secondary verbal memory were associated with functional outcomes in community/daily activity (e.g., going to school, working). Shortterm and secondary verbal memory were also largely associated with psychosocial skill acquisition. Furthermore, impairments in secondary verbal memory, vigilance, and to a lesser extent in executive function seemed to negatively affect social problemsolving skills. Composite scores show also a moderate to high association with the functional outcomes of the disease (86, 87). Hence, cognitive enhancement may have a significant impact on function, quality of life, patient well-being, as well as on the prevention of psychotic relapse (90). It is therefore recommended that the individual approach of each patient by healthcare professionals as well as the pharmacological treatment plan include the minimum necessary dose of antipsychotic medication, the restriction of polypharmacy, and caution in prescribing medicines with known anticholinergic activity. Finally, clinicians are required to reconsider the need for anticholinergic treatment before recommending any method or treatment for cognitive rehabilitation in schizophrenia.

\section{Quality Assessment and Risk of Bias}

One of the main limitations of the studies included is the small sample size of the participants. Furthermore, amongst prospective cohort studies $(48,49)$, the lack of a control group makes the evaluation environment an important confounding 
factor and limits the interpretation of the results. Participant groups were matched by at least age and one other factor as well as in the studies without control group, a range of factors such as age, gender, education, and symptom severity were considered covariate adjustments in analysis. However, possible confounding factors such as the indirect treatment positive effect on cognition, medication adherence, and impact of other neurotransmitter systems have not been adjusted in most studies included. In addition, the inpatient setting in several studies could also affect the cognitive and daily function outcome and hasn't considered as a confounder factor $(39,40,42,44,49,50$, 52). Regarding the included clinical studies of the systematic review $(52,53)$, they are characterized by low methodological quality with deficiencies mainly in terms of randomization and blinding methodology.

\section{Limitations}

In terms of the present systematic review limitations, no metaanalysis could be performed due to the heterogeneity in the populations, methodology, and interventions of the included studies. Therefore, this affects the quality of the evidence presented because it is unclear whether the positive studies were favorable or whether there was a performance bias. Language bias is also possible as we could have missed non-English studies. Moreover, according to the hierarchy of evidence, most of the studies included on their methodological design are cross-sectional or retrospective studies with a limited number of participants. Hence, there is no time sequence between the exposure and the disease-outcome studied and cannot safely prove a causal relationship.

It is also worth mentioning that the studies included were based on tools which vary in terms of the classification of the anticholinergic burden of active substances, and which also have several limitations. Although the method of measuring SAA is the gold standard method for assessing anticholinergicity, this may reflect only a transitional cholinergic condition outside the brain, which confers an intuitive clinical capability but lacks a direct in vivo assessment of the central effect of anticholinergic medication. Furthermore, as a tool it cannot be used to draw conclusions about which medicine in particular may need to be discontinued to reduce the anticholinergic load, while the cost

\section{REFERENCES}

1. Raedler TJ, Bymaster FP, Tandon R, Copolov D, Dean B. Towards a muscarinic hypothesis of schizophrenia. Mol Psychiatry. (2007) 12:23246. doi: 10.1038/sj.mp.4001924

2. Garcia-Rill E, Biedermann JA, Chambers T, Skinner RD, Mrak RE, Husain M, et al. Mesopontine neurons in schizophrenia. Neuroscience. (1995) 66:32135. doi: 10.1016/0306-4522(94)00564-L

3. Deng C, Huang X-F. Decreased density of muscarinic receptors in the superior temporal gyrusin schizophrenia. J Neurosci Res. (2005) 81:88390. doi: 10.1002/jnr.20600

4. Raedler TJ, Knable MB, Jones DW, Urbina RA, Gorey JG, Lee $\mathrm{KS}$, et al. In vivo determination of muscarinic acetylcholine receptor availability in schizophrenia. Am J Psychiatry. (2003) 160:118-27. doi: 10.1176/appi.ajp.160.1.118 and availability of the method lead to the use of anticholinergic drug scales, which also have certain limitations $(27,41)$. In general, anticholinergic drug scales cannot calculate systemic drug exposure, brain delivery, and distribution of drugs or drug interactions that can often affect overall anticholinergic activity. Moreover, although the scales do not differ greatly in the classification of most medicines, discrepancies that could affect the outcome have been observed (e.g., in the case of quetiapine) $(91,92)$.

\section{Conclusion}

The present systematic review shows that medication with increased anticholinergic load is possible to affect the cognitive functions of people with schizophrenia. However, based on different research methodologies and the clinical heterogeneity among various studies, it is not reasonable to make a definitive conclusion. Well-designed large prospective studies and randomized clinical trials are required to examine the effect of anticholinergic drug treatment on cognition in patients with schizophrenia. Based on these findings, clinicians are required to reconsider the need for anticholinergic treatment, with caution in prescribing medicines with known anticholinergic activity, before recommending any treatment for cognitive rehabilitation in schizophrenia.

\section{DATA AVAILABILITY STATEMENT}

The original contributions presented in the study are included in the article/supplementary material, further inquiries can be directed to the corresponding author/s.

\section{AUTHOR CONTRIBUTIONS}

RG carried out literature searches, appraised the articles, summarized the results, prepared the tables and figures, wrote the manuscript, and interpreted the results. DL interpreted the results. KG supervised the process, carried out literature searches, appraised the articles, summarized the results, wrote the manuscript, and interpreted the results. All authors contributed to the article and approved the submitted version.
5. Scarr E, Dean B. Role of the cholinergic system in the pathology and treatment of schizophrenia. Expert Rev Neurother. (2009) 9:7386. doi: 10.1586/14737175.9.1.73

6. Bakker G, Vingerhoets C, Boucherie D, Caan M, Bloemen O, Eersels J, et al. Relationship between muscarinic M1 receptor binding and cognition in medication-free subjects with psychosis. NeuroImage Clin. (2018) 18:7139. doi: 10.1016/j.nicl.2018.02.030

7. Martin-Ruiz CM, Haroutunian VH, Long P, Young AH, Davis KL, Perry EK, et al. Dementia rating and nicotinic receptor expression in the prefrontal cortex in schizophrenia. Biol Psychiatry. (2003) 54:122233. doi: 10.1016/S0006-3223(03)00348-2

8. Marutle A, Zhang X, Court J, Piggott M, Johnson M, Perry $\mathrm{R}$, et al. Laminar distribution of nicotinic receptor subtypes in cortical regions in schizophrenia. J Chem Neuroanat. (2001) 22:115-26. doi: 10.1016/S0891-0618(01)00117-X 
9. Stefansson H, Rujescu D, Cichon S, Pietiläinen $\mathrm{OPH}$, Ingason A, Steinberg S, et al. Large recurrent microdeletions associated with schizophrenia. Nature. (2008) 455:232-6. doi: 10.1038/nature07229

10. Adams CE, Stevens KE. Evidence for a role of nicotinic acetylcholine receptors in schizophrenia. Front Biosci. (2007) 12:4755-72. doi: 10.2741/2424

11. D'Souza MS, Markou A. Schizophrenia and tobacco smoking comorbidity: NAChR agonists in the treatment of schizophreniaassociated cognitive deficits. Neuropharmacology. (2012) 62:156473. doi: 10.1016/j.neuropharm.2011.01.044

12. Moore H, Fadel J, Sarter M, Bruno JP. Role of accumbens and cortical dopamine receptors in the regulation of cortical acetylcholine release. Neuroscience. (1999) 88:811-22. doi: 10.1016/S0306-4522(98)00261-9

13. Neigh GN, Arnold HM, Rabenstein RL, Sarter M, Bruno JP. Neuronal activity in the nucleus accumbens is necessary for performance-related increases in cortical acetylcholine release. Neuroscience. (2004) 123:63545. doi: 10.1016/j.neuroscience.2003.10.006

14. Zmarowski A, Sarter M, Bruno JP. NMDA and dopamine interactions in the nucleus accumbens modulate cortical acetylcholine release. Eur J Neurosci. (2005) 22:1731-40. doi: 10.1111/j.1460-9568.2005.04333.x

15. Kozak R, Martinez V, Young D, Brown H, Bruno JP, Sarter M. Toward a neuro-cognitive animal model of the cognitive symptoms of schizophrenia: disruption of cortical cholinergic neurotransmission following repeated amphetamine exposure in attentional task-performing, but not non-performing, rats. Neuropsychopharmacology. (2007) 32:207486. doi: 10.1038/sj.npp.1301352

16. St. Peters M, Demeter E, Lustig C, Bruno JP, Sarter M. Enhanced control of attention by stimulating mesolimbic-corticopetal cholinergic circuitry. $J$ Neurosci. (2011) 31:9760-71. doi: 10.1523/JNEUROSCI.1902-11.2011

17. D'Souza RS, Hooten WM. Extrapyramidal Symptoms. In: StatPearls [Internet]. Treasure Island, FL: StatPearls Publishing (2021). Available online at: https://www.ncbi.nlm.nih.gov/books/NBK534115/

18. Frankenburg FR. Schizophrenia Medication: Antipsychotics, 1st Generation, Antipsychotics, 2nd Generation, Serotonin-Dopamine Activity Modulators. (2020).

19. Rehse M, Bartolovic M, Baum K, Richter D, Weisbrod M, RoeschEly D. Influence of antipsychotic and anticholinergic loads on cognitive functions in patients with schizophrenia. Schizophr Res Treat. (2016) 2016:8213165. doi: 10.1155/2016/8213165

20. Elie D, Poirier M, Chianetta J, Durand M, Grégoire C, Grignon S. Cognitive effects of antipsychotic dosage and polypharmacy: a study with the BACS in patients with schizophrenia and schizoaffective disorder. J Psychopharmacol. (2010) 24:1037-44. doi: 10.1177/0269881108100777

21. Ritsner MS. Polypharmacy in Psychiatry Practice, Volume I: Multiple Medication Use. Dordrecht: Springer (2013). doi: 10.1007/978-94-007-5799-8

22. Tani M, Akashi N, Hori K, Konishi K, Kitajima Y, Tomioka H, et al. Anticholinergic activity and schizophrenia. Neurodegener Dis. (2015) 15:16874. doi: 10.1159/000381523

23. Fett AKJ, Viechtbauer W, Dominguez $M$ de G, Penn DL, van Os J, Krabbendam $\mathrm{L}$. The relationship between neurocognition and social cognition with functional outcomes in schizophrenia: a meta-analysis. Neurosci Biobehav Rev. (2011) 35:573-88. doi: 10.1016/j.neubiorev.2010.07.001

24. Green MF, Kern RS, Heaton RK. Longitudinal studies of cognition and functional outcome in schizophrenia: implications for MATRICS. Schizophr Res. (2004) 72:41-51. doi: 10.1016/j.schres.2004.09.009

25. Moher D, Liberati A, Tetzlaff J, Altman DG. Preferred reporting items for systematic reviews and meta-analyses: the PRISMA statement. Int J Surg. (2010) 8:336-41. doi: 10.1016/j.ijsu.2010.02.007

26. O'Connor DA, Green SE, Higgins JPT. Defining the review question and developing criteria for including studies. In: Higgins JPT, Green S, editors. Cochrane Handbook for Systematic Reviews of Interventions. 1st ed. London: John Wiley \& Sons (2008). p. 83-94.

27. Carnahan RM, Lund BC, Perry PJ, Pollock BG, Gulp KR. The anticholinergic drug scale as a measure of drug-related anticholinergic burden: associations with serum anticholinergic activity. J Clin Pharmacol. (2006) 46:14816. doi: 10.1177/0091270006292126

28. Ruxton K, Woodman RJ, Mangoni AA. Drugs with anticholinergic effects and cognitive impairment, falls and all-cause mortality in older adults: a systematic review and meta-analysis. Br J Clin Pharmacol. (2015) 80:20920. doi: $10.1111 /$ bcp. 12617

29. Boustani M, Campbell N, Munger S, Maidment I, Fox C. Impact of anticholinergics on the aging brain: a review and practical application. Aging Health. (2008) 4:311-20. doi: 10.2217/1745509X.4.3.311

30. Ancelin ML, Artero S, Portet F, Dupuy AM, Touchon J, Ritchie K. Nondegenerative mild cognitive impairment in elderly people and use of anticholinergic drugs: longitudinal cohort study. Br Med J. (2006) 332:4558. doi: 10.1136/bmj.38740.439664.DE

31. Tune L, Coyle JT. Serum levels of anticholinergic drugs in treatment of acute extrapyramidal side effects. Arch Gen Psychiatry. (1980) 37:2937. doi: 10.1001/archpsyc.1980.01780160063007

32. Nuechterlein KH, Green MF, Kern RS, Baade LE, Barch DM, Cohen JD, et al. The MATRICS consensus cognitive battery, part 1: test selection, reliability, and validity. Am J Psychiatry. (2008) 165:203-13. doi: 10.1176/appi.ajp.2007.0701 0042

33. Keefe RSE, Fenton WS. How should DSM-V criteria for schizophrenia include cognitive impairment? Schizophr Bull. (2007) 34:912-20. doi: 10.1093/schbul/sbm046

34. Stang A. Critical evaluation of the newcastle-ottawa scale for the assessment of the quality of nonrandomized studies in meta-analyses. Euro J Epidemiol. (2010) 25:603-5. doi: 10.1007/s10654-010-9491-z

35. Modesti PA, Reboldi G, Cappuccio FP, Agyemang C, Remuzzi G, Rapi S, et al. Panethnic differences in blood pressure in Europe: a systematic review and meta-analysis. PLoS ONE. (2016) 11:e0417601. doi: 10.1371/journal.pone.0147601

36. Jadad AR, Moore RA, Carroll D, Jenkinson C, Reynolds DJM, Gavaghan DJ, et al. Assessing the quality of reports of randomized clinical trials: is blinding necessary? Control Clin Trials. (1996) 17:1-12. doi: 10.1016/0197-2456(95)00134-4

37. Oremus M, Wolfson C, Perrault A, Demers L, Momoli F, Moride Y. Interrater reliability of the modified jadad quality scale for systematic reviews of alzheimer's disease drug trials. Dement Geriatr Cogn Disord. (2001) 12:2326. doi: 10.1159/000051263

38. Ang MS, Abdul Rashid NA, Lam M, Rapisarda A, Kraus M, Keefe RSE, et al. The impact of medication anticholinergic burden on cognitive performance in people with schizophrenia. J Clin Psychopharmacol. (2017) 37:6516. doi: 10.1097/JCP.0000000000000790

39. Eum S, Hill SK, Rubin LH, Carnahan RM, Reilly JL, Ivleva I, et al. Cognitive burden of anticholinergic medications in psychotic disorders. Schizophr Res. (2018) 190:129-35. doi: 10.1016/j.schres.2017.03.034

40. Kim SJ, Jung D, Shim JC, Moon JJ, Jeon DW, Kim YN, et al. The effect of anticholinergic burden on cognitive and daily living functions in patients with schizophrenia. Asian J Psychiatr. (2019) 46:1117. doi: 10.1016/j.ajp.2019.10.013

41. Minzenberg MJ, Poole JH, Benton C, Vinogradov S. Assocation of anticholinergic load with impairment of complex attention and memory in schizophrenia. Am J Psychiatry. (2004) 161:11624. doi: 10.1176/appi.ajp.161.1.116

42. Perlick D, Stastny P, Katz I, Mayer M, Mattis S. Memory deficits and anticholinergic levels in chronic schizophrenia. Am J Psychiatry. (1986) 143:230-2. doi: 10.1176/ajp.143.2.230

43. Sweeney JA, Keilp JG, Haas GL, Hill J, Weiden PJ. Relationships between medication treatments and neuropsychological test performance in schizophrenia. Psychiatry Res. (1991) 37:297308. doi: 10.1016/0165-1781(91)90065-W

44. Tracy JI, Monaco C, Giovannetti T, Abraham G, Josiassen RC. Anticholinergicity and cognitive processing in chronic schizophrenia. Biol Psychol. (2001) 56:1-22. doi: 10.1016/S0301-0511(00)00083-1

45. Tsoutsoulas C, Mulsant BH, Kumar S, Ghazala Z, Voineskos $\mathrm{AN}$, Menon $\mathrm{M}$, et al. Anticholinergic burden and cognition in older patients with schizophrenia. J Clin Psychiatry. (2017) 78:e1284-90. doi: 10.4088/JCP.17m11523

46. Tune LE, Strauss ME, Lew MF, Breitlinger E, Coyle JT. Serum levels of anticholinergic drugs and impaired recent memory in chronic schizophrenic patients. Am J Psychiatry. (1982) 139:1460-2. doi: 10.1176/ajp.139.11.1460 
47. Joshi YB, Thomas ML, Braff DL, et al. Anticholinergic medication burdenassociated cognitive impairment in schizophrenia. Am J Psychiatry. (2021) 178:838-47. doi: 10.1176/appi.ajp.2020.20081212

48. Desmarais JE, Beauclair L, Annable L, Bélanger MC, Kolivakis TT, Margolese HC. Effects of discontinuing anticholinergic treatment on movement disorders, cognition and psychopathology in patients with schizophrenia. Ther Adv Psychopharmacol. (2014) 4:257-67. doi: 10.1177/2045125314553611

49. Drimer T, Shahal B, Barak Y. Effects of discontinuation of longterm anticholinergic treatment in elderly schizophrenia patients. Int Clin Psychopharmacol. (2004) 19:27-9. doi: 10.1097/00004850-200401000-00005

50. Ogino S, Miyamoto S, Tenjin T, Kitajima R, Ojima K, Miyake N, et al. Effects of discontinuation of long-term biperiden use on cognitive function and quality of life in schizophrenia. Prog Neuro-Psychopharmacol Biol Psychiatry. (2011) 35:78-83. doi: 10.1016/j.pnpbp.2010.08.030

51. Tracy JI, Monaco CA, Abraham G, Josiassen RC, Pollock BG. Relation of serum anticholinergicity to cognitive status in schizophrenia patients taking clozapine or risperidone. J Clin Psychiatry. (1998) 59:184-8. doi: 10.4088/JCP.v59n0407

52. Hitri A, Craft RB, Sethi R, Sinha D. Drug levels and antiparkinsonian drugs in neuroleptic-treated schizophrenic patients. Clin Neuropharmacol. (1987) 10:261-71. doi: 10.1097/00002826-198706000-00007

53. Vinogradov S, Fisher M, Warm H, Holland C, Kirshner MA, Pollock BG. The cognitive cost of anticholinergic burden: decreased response to cognitive training in schizophrenia. Am J Psychiatry. (2009) 166:105562. doi: 10.1176/appi.ajp.2009.09010017

54. Newman EL, Gupta K, Climer JR, Monaghan CK, Hasselmo ME. Cholinergic modulation of cognitive processing: insights drawn from computational models. Front Behav Neurosci. (2012) 6:24. doi: 10.3389/fnbeh.2012.00024

55. Bowie CR, Harvey PD. Cognitive deficits and functional outcome in schizophrenia. Neuropsychiatric Dis Treat. (2006) 2:531-6. doi: 10.2147/nedt.2006.2.4.531

56. Keefe RSE, Silva SG, Perkins DO, Lieberman JA. The effects of atypical antipsychotic drugs on neurocognitive impairment in schizophrenia: a review and meta-analysis. Schizophr Bull. (1999) 25:201-22. doi: 10.1093/oxfordjournals.schbul.a033374

57. Fujimaki K, Takahashi T, Morinobu S. Association of typical versus atypical antipsychotics with symptoms and quality of life in schizophrenia. PLOS ONE. (2012) 7:e0037087. doi: 10.1371/journal.pone.0037087

58. Tani H, Takasu S, Uchida H, Suzuki T, Mimura M, Takeuchi H. Factors associated with successful antipsychotic dose reduction in schizophrenia: a systematic review of prospective clinical trials and meta-analysis of randomized controlled trials. Neuropsychopharmacology. (2020) 45:887. doi: 10.1038/s41386-019-0573-7

59. Cools R, D'Esposito. Inverted-U-shaped dopamine actions on human working memory and cognitive control. Biol Psychiatry. (2011) 69:e11325. doi: 10.1016/j.biopsych.2011.03.028

60. Manschreck TC, Redmond DA, Candela SF, Maher BA. Effects of clozapine on psychiatric symptoms, cognition, and functional outcome in schizophrenia. $J$ Neuropsychiatry Clin Neurosci. (1999) 11:481-9. doi: 10.1176/jnp.11.4.481

61. McGurk SR. The effects of clozapine on cognitive functioning in schizophrenia. J Clin Psychiatry. (1999) 60:24-9.

62. Meltzer HY, Park S, Kessler R. Cognition, schizophrenia, and the atypical antipsychotic drugs. Proc Natl Acad Sci USA. (1999) 96:135913. doi: 10.1073 /pnas.96.24.13591

63. Costa-Dookhan KA, Agarwal SM, Chintoh A, Tran VN, Stogios N, Ebdrup $\mathrm{BH}$, et al. The clozapine to norclozapine ratio: a narrative review of the clinical utility to minimize metabolic risk and enhance clozapine efficacy. Expert Opin Drug Saf. (2020) 19:43-57. doi: 10.1080/14740338.2020.1698545

64. Park R, Kim S, Kim E. Relationship of change in plasma clozapine/n-desmethylclozapine ratio with cognitive performance in patients with schizophrenia. Psychiatry Investig. (2020) 17:1158-65. doi: 10.30773/pi.2020.0306

65. Couchman L, Bowskill SV, Handley S, Patel MX, Flanagan RJ. Plasma clozapine, norclozapine, and the clozapine:norclozapine ratio in relation to prescribed dose and other factors: data from a therapeutic drug monitoring service, 1993-2007. Ther Drug Monit. (2010) 32:438-47. doi: 10.1097/FTD.0b013e3181 dad1fb
66. de Leon J. Atypical antipsychotic dosing: the effect of smoking and caffeine. Psychiatr Serv. (2004) 55:491-3. doi: 10.1176/appi.ps.55.5.491

67. Grover S, Chakrabarti S, Kulhara P, Avasthi A. Clinical practice guidelines for management of schizophrenia. Indian J Psychiatry. (2017) 59:S1933. doi: 10.4103/0019-5545.196972

68. WHO Mental Health Gap Action. WHO |Role of Anticholinergic Medications in Patients Requiring Long-Term Antipsychotic Treatment for Psychotic Disorders. WHO (2015). Available online at: http://www.who.int/mental_ health/mhgap/evidence/psychosis/q6/en/

69. Bergendal A, Schiöler H, Wettermark B, Björkstén KS. Concomitant use of two or more antipsychotic drugs is common in Sweden. Ther Adv Psychopharmacol. (2015) 5:224-31. doi: 10.1177/2045125315588647

70. Broekema WJ, De Groot AIW, Van Harten APN. Simultaneous prescribing of atypical antipsychotics, conventional antipsychotics and anticholinergics-a European study. Pharm World Sci. (2007) 29:126-30. doi: 10.1007/s11096-006-9063-1

71. Gallego JA, Bonetti J, Zhang J, Kane JM. Prevalence and correlates of antipsychotic polypharmacy: a systematic review and meta-regression of global and regional trends from the 1970s to 2009. Schizophr Res. (2012) 138:18-28. doi: 10.1016/j.schres.2012.03.018

72. Magliano L, Fiorillo A, Guarneri M, Marasco C, De Rosa C, Malangone C, et al. Prescription of psychotropic drugs to patients with schizophrenia: an Italian national survey. Eur J Clin Pharmacol. (2004) 60:513-22. doi: 10.1007/s00228-004-0803-7

73. Su YA, Yan F, Li Q, Xiang YT, Shu L, Yu X, et al. Anticholinergic use trends in 14,013 patients with schizophrenia from three national surveys on the use of psychotropic medications in China (2002-2012). Psychiatry Res. (2017) 257:132-6. doi: 10.1016/j.psychres.2017.07.038

74. Chakos MH, Glick ID, Miller AL, Hamner MB, Miller DD, Patel JK, et al. Special section on CATIE baseline data: baseline use of concomitant psychotropic medications to treat schizophrenia in the CATIE trial. Psychiatr Serv. (2006) 57:1094-101. doi: 10.1176/ps.2006.57.8.1094

75. Correll CU, Gallego JA. Antipsychotic polypharmacy. A comprehensive evaluation of relevant correlates of a long-standing clinical practice. Psychiatr Clin North Am. (2012) 35:661-81. doi: 10.1016/j.psc.2012.06.007

76. Feigenson KA, Kusnecov AW, Silverstein SM. Inflammation and the twohit hypothesis of schizophrenia. Neurosci Biobehav Rev. (2014) 38:7293. doi: 10.1016/j.neubiorev.2013.11.006

77. Insel TR. Rethinking schizophrenia. Nature. (2010) 468:18793. doi: $10.1038 /$ nature 09552

78. Pittman-Polletta BR, Kocsis B, Vijayan S, Whittington MA, Kopell NJ. Brain rhythms connect impaired inhibition to altered cognition in schizophrenia. Biol Psychiatry. (2015) 77:1020-30. doi: 10.1016/j.biopsych.2015.02.005

79. Hsu WY, Lane HY, Lin CH. Medications used for cognitive enhancement in patients with schizophrenia, bipolar disorder, Alzheimer's disease, and Parkinson's disease. Front Psychiatry. (2018) 9:91. doi: 10.3389/fpsyt.2018.00091

80. Keefe RSE. Why are there no approved treatments for cognitive impairment in schizophrenia? World Psychiatry. (2019) 18:167-8. doi: 10.1002/wps.20648

81. Pae C-U. Role of the cholinesterase inhibitors in the treatment of schizophrenia. Expert Opin Investig Drugs. (2013) 22:2938. doi: $10.1517 / 13543784.2013 .762355$

82. Singh J, Kour K, Jayaram MB. Acetylcholinesterase inhibitors for schizophrenia. Cochrane Database Syst Rev. (2012) 1:CD007967. doi: 10.1002/14651858.CD007967.pub2

83. Guercio GD, Thomas ME, Cisneros-Franco JM, Voss P, Panizzutti R, de Villers-Sidani E. Improving cognitive training for schizophrenia using neuroplasticity enhancers: lessons from decades of basic and clinical research. Schizophr Res. (2019) 207:80-92. doi: 10.1016/j.schres.2018.04.028

84. Fisher M, Holland C, Merzenich MM, Vinogradov S. Using neuroplasticitybased auditory training to improve verbal memory in schizophrenia. Am J Psychiatry. (2009) 166:805-11. doi: 10.1176/appi.ajp.2009.080 50757

85. Fisher M, Loewy R, Carter C, Lee A, Ragland JD, Niendam T, et al. Neuroplasticity-Based auditory training via laptop computer improves cognition in young individuals with recent onset schizophrenia. Schizophr Bull. (2015) 41:250-8. doi: 10.1093/schbul/sbt232 
86. Green MF. What are the functional consequences of neurocognitive deficits in schizophrenia?. Am J Psychiatry. (1996) 153:32130. doi: 10.1176/ajp.153.3.321

87. Green MF, Kern RS, Braff DL, Mintz J. Neurocognitive deficits and functional outcome in schizophrenia: are we measuring the "right stuff"? Schizophr Bull. (2000) 126:119-36. doi: 10.1093/oxfordjournals.schbul. a033430

88. Velligan DI, Mahurin RK, Diamond PL, Hazleton BC, Eckert SL, Miller AL. The functional significance of symptomatology and cognitive function in schizophrenia. Schizophr Res. (1997) 25:21-31. doi: 10.1016/S0920-9964(97)00010-8

89. Jääskeläinen E, Juola P, Hirvonen N, Mcgrath JJ, Saha S, Isohanni $\mathrm{M}$, et al. A systematic review and meta-analysis of recovery in schizophrenia. Schizophr Bull. (2013) 39:1296-306. doi: 10.1093/schbul/ sbs 130

90. Sahakian BJ, Savulich G. Innovative methods for improving cognition, motivation and wellbeing in schizophrenia. World Psychiatry. (2019) 18:16870. doi: 10.1002/wps.20649

91. López-Álvarez J, Sevilla-Llewellyn-Jones J, Agüera-Ortiz L. Anticholinergic drugs in geriatric psychopharmacology. Front Neurosci. (2019) 13:1309. doi: 10.3389/fnins.2019. 01309
92. Naples JG, Marcum ZA, Perera S, Gray SL, Newman AB, Simonsick EM, et al. Concordance between anticholinergic burden scales. J Am Geriatr Soc. (2015) 63:2120-4. doi: 10.1111/jgs.13647

Conflict of Interest: The authors declare that the research was conducted in the absence of any commercial or financial relationships that could be construed as a potential conflict of interest.

Publisher's Note: All claims expressed in this article are solely those of the authors and do not necessarily represent those of their affiliated organizations, or those of the publisher, the editors and the reviewers. Any product that may be evaluated in this article, or claim that may be made by its manufacturer, is not guaranteed or endorsed by the publisher.

Copyright (C) 2021 Georgiou, Lamnisos and Giannakou. This is an open-access article distributed under the terms of the Creative Commons Attribution License (CC BY). The use, distribution or reproduction in other forums is permitted, provided the original author(s) and the copyright owner(s) are credited and that the original publication in this journal is cited, in accordance with accepted academic practice. No use, distribution or reproduction is permitted which does not comply with these terms. 